\title{
Evaluation of a Nurse-led Intervention to Improve Palliative Care for Select Medical Intensive Care Patients
}

Lori A. Constantine

West Virginia University

Follow this and additional works at: https://researchrepository.wvu.edu/etd

\section{Recommended Citation}

Constantine, Lori A., "Evaluation of a Nurse-led Intervention to Improve Palliative Care for Select Medical Intensive Care Patients" (2013). Graduate Theses, Dissertations, and Problem Reports. 126.

https://researchrepository.wvu.edu/etd/126

This Dissertation is protected by copyright and/or related rights. It has been brought to you by the The Research Repository @ WVU with permission from the rights-holder(s). You are free to use this Dissertation in any way that is permitted by the copyright and related rights legislation that applies to your use. For other uses you must obtain permission from the rights-holder(s) directly, unless additional rights are indicated by a Creative Commons license in the record and/ or on the work itself. This Dissertation has been accepted for inclusion in WVU Graduate Theses, Dissertations, and Problem Reports collection by an authorized administrator of The Research Repository @ WVU.

For more information, please contact researchrepository@mail.wvu.edu. 


\title{
Evaluation of a Nurse-led Intervention to Improve Palliative Care for Select Medical Intensive Care Patients
}

\author{
Lori A. Constantine, MSN, FNP-BC \\ Doctoral Capstone Paper submitted \\ to the School of Nursing \\ West Virginia University \\ in partial fulfillment of the requirements for the degree of \\ Doctor of Nursing Practice
}

\author{
Gina Maiocco, PhD, RN, CCNS, Chair \\ Alvin Moss, MD, FACP, FAAHPM \\ Patty Hermosilla, DNP, FNP-BC
}

Department of Nursing

Morgantown, West Virginia

2013

Keywords: Nursing, Palliative Care, Intensive Care 


\title{
Abstract \\ Evaluation of a Nurse-led Intervention to Improve Palliative Care for Select Medical Intensive Care Patients
}

\author{
Lori A. Constantine
}

Body: Twenty percent of all Americans die in an intensive care unit (ICU) or shortly thereafter. For most of these patients, relentless and distressing symptoms are commonplace. Review of local data reveals opportunity for improvement in the palliative care offered to hospitalized patients. To address this need for improved palliative care, the Volunteer Hospital Association's (VHA) Communication and Care Bundle will be implemented in a local medical ICU, using Roger's (2003) diffusion of innovations (DOI) as the theoretical base.

Evaluation: A descriptive, retrospective design evaluated if a nurse-led, performance improvement project increased the percentage of time that nurses documented the nine quality measures of the Communication and Care Bundle within a given time frames, for select medical ICU patients. ICU length of stay and patient mortality were also assessed before and after the multifaceted intervention.

Results: The implementation of the Communication and Care Bundle led to significantly improved documentation of appropriate decision makers and advance directives by day one, and care management contact by day three. Do not resuscitate (DNR) preferences significantly increased in the post-intervention phase of this study. Surprisingly, optimal pain management significantly decreased in the post-intervention period. ICU lengths of stay and mortality results are questionable due to a change in inclusion criteria from the pre-intervention to the postintervention groups.

Discussion and Recommendations: The adoption of the Communication and Care Bundle has been shown to increase documentation of three of the nine quality measures in the medical intensive care unit (MICU) and surgical intensive care unit (SICU) for adult medical intensive care patients at high risk of death. Further inquiry is needed to fully examine why all quality measures did not significantly increase. 


\section{Acknowledgements}

I would like to sincerely thank my capstone committee for all of their help and support throughout this project. Gina Maiocco, Alvin Moss, and Patty Hermosilla have been invaluable mentors, educators, and clinicians. Their experience and leadership guided me through the completion of my capstone. I would also like to thank the Doctor of Nursing faculty of West Virginia University (WVU) that helped me through countless papers and presentations. I must also thank Emily Barnes, my advisor and friend, for her on and off the record advice, and many tissues. I must also acknowledge the members of WVU Healthcare Supportive Care Service whose passion for quality patient care continues to inspire me and push me to seek excellence in care for my patients.

Attainment of my Doctor in Nursing Practice would not have been possible if it was not for the unending support and love I received from my family. They often had to wait for dinner, deal with my stress, forgo play dates at the park, do extra chores, and skip many date nights while I pursued my education. While it was never easy, my family supported me and continually gave of themselves to make completion of my education a reality. Finally, I must thank God for placing me where I needed to be when I needed to be there. With Him, anything is possible. 


\section{Table of Contents}

$\begin{array}{ll}\text { Introduction } & 1\end{array}$

$\begin{array}{ll}\text { Background and Significance } & 1\end{array}$

Chronic Disease in West Virginia 1

Preferences for Care $\quad 1$

Misaligned Domains $\quad 3$

Cost to Society 4

$\begin{array}{ll}\text { Problem Statement } & 10\end{array}$

Definition of "High Risk of Death" 10

Communication and Care Bundle Processes 11

$\begin{array}{ll}\text { Theoretical Framework } & 11\end{array}$

$\begin{array}{ll}\text { Key Elements } & 12\end{array}$

Theory Validity to Proposed Project $\quad 14$

Theory Application to Current Project 15

$\begin{array}{ll}\text { Literature Review and Synthesis } & 18\end{array}$

$\begin{array}{ll}\text { Methodology } & 18\end{array}$

$\begin{array}{ll}\text { Critical Appraisal } & 19\end{array}$

$\begin{array}{ll}\text { Clinical Guidelines } & 20\end{array}$

$\begin{array}{ll}\text { Research Studies } & 21\end{array}$

$\begin{array}{ll}\text { Synthesis } & 35\end{array}$

Congruence of Organization’s Strategic Plan to Project 39

$\begin{array}{ll}\text { Project Description } & 40\end{array}$

$\begin{array}{ll}\text { The IPAL Project } & 40\end{array}$

The Communication and Care Bundle 40

Research Questions $\quad 43$ 
Project Objectives $\quad 43$

$\begin{array}{ll}\text { Project Design } & 45\end{array}$

Evidenced Based Project/Intervention Plan 45

Stakeholder Support $\quad 46$

$\begin{array}{ll}\text { Patient Population } & 47\end{array}$

$\begin{array}{ll}\text { Sampling Plan } & 49\end{array}$

$\begin{array}{lr}\text { Data Collection } & 50\end{array}$

Multifaceted Interventions to Increase Project Adoption 51

$\begin{array}{ll}\text { Resources } & 52\end{array}$

Evidence of Support $\quad 53$

$\begin{array}{lr}\text { Evaluation } & 53\end{array}$

$\begin{array}{ll}\text { Results } & 54\end{array}$

Quality Measure $\quad 54$

Patient Outcomes $\quad 56$

$\begin{array}{ll}\text { Patient Population } & 57\end{array}$

$\begin{array}{ll}\text { Nurse Demographics } & 57\end{array}$

$\begin{array}{lr}\text { Discussion } & 59\end{array}$

$\begin{array}{ll}\text { Limitations } & 62\end{array}$

$\begin{array}{ll}\text { Conclusions } & 66\end{array}$

Additional Findings and Recommendations $\quad 66$

$\begin{array}{ll}\text { Summary and Implications } & 67\end{array}$

$\begin{array}{lr}\text { Appendices } & 69\end{array}$

Appendix A: 
Appendix B:

Figure 2: Theory of Diffusion of Innovations "S-Shaped Curve"

Appendix C:

Table 1: Palliative Care Processes of the Communication and Care Bundle

Appendix D:

Figure 3: Supportive Care Patient Information Leaflet

Appendix E: Project Brief

Appendix F: Educational Outline

Appendix G:

Figure 4: Poster Displayed in MICU and SICU

Appendix H: Nurse Checklist

Appendix I: Pre-Intervention Inclusion Criteria

Appendix J: Post-Intervention Inclusion Criteria

Appendix K: Inclusion Criteria Change Approval Letter

Appendix L: Chart Abstraction Tool

Appendix M:

Table M1: Project Budget Personnel Costs

Table M2: Project Budget Equipment/Supplies Costs

Appendix P:

Table P1: Quality Measure Frequencies both Pre-Intervention and Post-Intervention as Documented by Day 1 
Table P2: Regular Pain Assessment and Optimal Pain Management Means both Pre-Intervention and Post-Intervention as Documented by Day 1

Table P3: Quality Measure Frequencies both Pre-Intervention and Post-Intervention as Documented by Day 3

Table P4: Quality Measure Frequencies both Pre-Intervention and Post-Intervention as Documented by Day 5

Table P5: Patient Outcome ICU Length of Stay both Pre-Intervention and Post-Intervention

Table P6: Patient Outcome Mortality both Pre-Intervention and PostIntervention

Table P7: Patient Population with the Specified Inclusion Criteria both Pre-Intervention and Post-Intervention

Table P8: Nurse Demographics Obtained During On-Line Educational Module

Table P9: Nurse Perspectives Related to Palliative Care in the ICU 


\section{Evaluation of a Nurse-led Intervention to Improve Palliative Care}

for Select Medical Intensive Care Patients

Twenty percent of all Americans die in an intensive care unit (ICU) or shortly after an ICU stay (Angus et al., 2004). For most of these patients, relentless and distressing symptoms are commonplace (Puntillo et al., 2010). This capstone proposal describes the implementation and evaluation of a nurse-led performance improvement project to integrate a bundle of palliative care processes into the care of medical ICU patients at high risk of death in northern WV. The theoretical framework, diffusion of innovation (DOI) (Rogers, 2003), guided the project implementation.

\section{Background and Significance}

\section{Chronic Disease}

West Virginians are plagued with a high mortality rate from chronic disease. Nearly 1.3 million cases of seven common chronic diseases (cancers, diabetes, heart disease, hypertension, stroke, mental disorders, and pulmonary conditions) were reported in West Virginia (WV) in 2003 (Milken Institute, 2007). In 2009, heart disease, cancer, and chronic lower respiratory diseases were the three leading causes of death in WV, accounting for $53.3 \%$ of WV resident deaths that year [WV Department of Health and Human Services (DHHS), 2009]. On its current trajectory, West Virginians will experience a dramatic increase in chronic disease in the next 20 years. Given the expected rise in life expectancy, these patients will occupy an increasing number of the acute care beds as patient disease states worsen. This coupled with increasing life expectancies will increase the total Medicare costs dramatically.

\section{Preferences for Care}


Although $75 \%$ to $90 \%$ of patients admitted to an ICU will survive to discharge (Luce \& Prendergast, 2001), 10 to $25 \%$ will not. Because the ICU by its nature is prone to have a higher mortality rate among its patients, many critical care organizations including the American Thoracic Society (Lankin et al., 2008), the American College of Chest Physicians (Selecky et al., 2005), and American College of Critical Care Medicine (Truog et al, 2008) have answered the call to critical care reform through critical care consensus and position statements for healthcare providers in this specialty area.

Nationally, $70 \%$ of the chronically ill patient population has said they would prefer to die at home (Cloud, 2000). Moreover, greater than $80 \%$ of patients with chronic diseases have expressed their desire to avoid hospitalization, especially the ICU when they are dying (Dartmouth Atlas of Health Care, 2011). Still, in the US, 53\% of patients die in hospitals and 24\% die in nursing homes [Center to Advance Palliative Care (CAPC), 2013]. Of the 53\% of hospital deaths, $20 \%$ of these patients will die in an ICU or shortly after an ICU stay (Angus et al., 2004). Similarly, when West Virginians were asked about end-of-life care options, twothirds preferred to forgo expensive end-of-life care in the hospital and wished to experience death in their own home (WV Center for End of Life Care, 2013). Yet, in 2005, 43\% of West Virginians died in hospitals (WV Department of Health and Human Services (WV DHHS, 2010).

When patients are hospitalized due to a health-related crisis their whole being is affected. Hospitalized patients are often forced to think about their own mortality. For the seriously ill, patients and families are often faced with making difficult decisions about future care. Palliative care can help hospitalized patients and their families deal with the stress of this acute assault. Palliative care addresses all aspects of life, not just the illness. Psychological, emotional, and 
spiritual wellness is as important as pain and symptom control, disease management, treatment, and cure if possible. Many falsely believe that palliative care simply equates to nearing death. However, aggressive treatment of disease and palliative care is often simultaneous in hospitals (see Appendix A, Figure 1). Palliative care helps patients cope with the human response to disease and illness, while focusing on patients' and families' goals of care, whatever they may be.

\section{Misaligned Domains}

In 1996, the ground-breaking Study to Understand Prognoses and Preferences for Outcomes and Risks of Treatments (SUPPORT) (1996) opened the country's eyes to the poor quality of care at the end-of-life in many hospitals; exposing dying patients wrought with uncontrolled pain and continued suffering. In phase II of this large $(n=4,804)$, randomized control trial (RCT), a nurse-led intervention in the ICU, focusing on improved communications with physician groups, patients, and families failed to change patient outcomes. A nurse trained in palliative care initiated communications with ICU physician groups regarding six-month prognosis estimates of their patients, outcomes of CPR, and predicted functional disability of their patients at two months. The nurse also initiated contacts with the patient, family, physician, and hospital staff to determine patient and family preferences, discuss patient outcomes, highlight pain control, discuss end of life options, and facilitate family meetings. Although, the intervention was not successful in changing patients' outcomes, the study did reveal the need for future research and interventions within this patient population.

Since then, there have been many initiatives and research-based projects aimed at improving end-of-life care in the ICU. Clarke et al. (2003) defined domains of quality for endof-life care and addressed how these domains guide potential quality improvement efforts 
focused on end-of-life care in the ICU. The domains include: patient and family-centered decision making, communication, continuity of care, emotional and practical support, symptom management and comfort care, spiritual support, and emotional and organizational support for ICU clinicians.

Currently the national system of healthcare is misaligned with the domains proposed by Clarke et al. (2003). Palliative care is not emphasized in our nation's medical and nursing schools (Ahmed, 2004). The deficiency of palliative care education is extensive. It occurs at the undergraduate and graduate level, as well post-graduation continuing education. Physicians and nurses both report this lack of education as a key factor in limiting their ability to provide quality palliative care for their patients (Boyd, Merkh, Rutledge, \& Randall, 2011; Ngo-Metzger, August, Srinivasan, Liao, \& Meyskens, 2008). Consequently, physicians and nurses do not feel comfortable with end-of-life discussions and are not sufficiently prepared to meet the end-of-life needs of the public (Barnett, 2002; Chiplasky, 2013). The majority of physicians overestimate prognoses and patients themselves often do not recognize that they are dying until very late [Institute for Clinical Systems Improvement (ICSI), 2011]. This leads to palliative discussions and consults being offered very late in the patient's disease trajectory (Bradley, Prigerson, Cherlin, Johnson-Hurzeler, \& Kasi, 2002), leading to increased hospitalizations and procedures that often do not benefit the patient (Morrison, Dietrich, Ladwig, Quill, Sacco, Tangeman, \& Meier, 2011).

\section{Cost to Society}

Financial costs. Hospital palliative care consultation teams have been associated with considerable hospital financial savings (Morrison et al., 2011). Currently, palliative or supportive care services are present at about half of the hospitals within the United States. 
Estimated savings are based upon a dispersion rate of palliative and supportive services to approximately $1.5 \%$ of all hospitalized patients. This represents a potential cost savings equal to approximately $\$ 1.2$ billion per year. If palliative or supportive care services were offered at greater than $90 \%$ of all U.S. hospitals, then direct cost savings would be between 4 and 6 billion dollars per year (Morrison, 2009). Furthermore, if palliative services were then utilized with 5\% to $7.5 \%$ of the hospital population, there is a predicted cost savings of $\$ 60$ billion dollars over a ten year time span (Morrison, 2009).

Research has shown that medical ICU stays can be significantly shortened (8.96 vs. 16.28 days, $\mathrm{p}<.0001$ ) by a proactive intervention of a palliative consult for ICU patients at high risk of death, without increasing mortality (Norton, Hogan, Holloway, Temkin-Greener, Buckley, \& Quill, 2007). According to Candrilli \& Mauskopf (2006), the mean cost of stay in the ICU per day by level of care across all conditions is approximately $\$ 2,401.00$. If these numbers are true, ICUs could sustain an average cost savings $\$ 17,575.32$ per patient by intervening in high risk patients early.

Global impact. Little is known about the quality of death and dying globally (Singer \& Bowman, 2002). Non-communicable diseases such as cardiovascular diseases, cancer, chronic respiratory diseases, and diabetes account for 36 million or $63 \%$ of global deaths annually (Demaio, 2012). Nearly $85 \%$ of the 56 million deaths worldwide that occur each year are in developing countries (Demaio, 2012). Recently, modernized countries have developed an appreciation for the need to improve palliative care. Emerging technological developments have made healthcare providers capable of significantly extending life, sometimes without regard to individual preferences (Singer \& Bowman, 2002). For example, simple cardiopulmonary resuscitation (CPR) orders are often not explored upon admission to the ICU (Cook et al., 2001). 
In a review of 2,916 adult patients from 15 ICUs in four countries, researchers reported that CPR orders were rarely established within 24 hours of admission to the ICU. This study has been replicated many times and is only one example of the barriers for effective palliative care in developing countries.

There is little research regarding palliative care in under-developed countries. However, inferences can be made. For example, in most of the world the majority of persons presenting with cancer are already in an advanced stage (Demaio, 2012). Hence, the only realistic treatment for many persons in under-developed countries is pain relief and palliative care. Furthermore, due to the scarcity of resources in these countries, palliative care may often be subpar or absent. Yet, these deficiencies of resources that may impede quality palliative care as experienced in modernized countries also empower families to play a larger role in the dying process of their loved ones.

National impact. Since the release of the original SUPPORT study, many other investigators have substantiated this study's concerning results (Cook, Guyatt, \& Rocker, 2001; Curt \& Rubenfeld, 2005; Desbiens, Wu, \& Broste, 1996; Nelson, Meier, and Oei, 2001; Puntillo et al. 2010, Stein-Parbury \& McKinley, 2000; Teno, Fisher, \& Hamel, 2000). Most recently, Puntillo et al. (2010) conducted brief surveys of 171 critically-ill patients to examine the prevalence, intensity, and related distress of ten common symptoms in the ICU. The most frequently reported symptoms were tiredness (75\%), thirst (71\%), and anxiety (58\%), with thirst rated as the most intense. The symptoms causing the most distress were shortness of breath, pain, and feeling scared or confused.

Another major national barrier to quality palliative care is that families fail to understand basic information about diagnosis, prognosis, or critical care treatments. Azoulay et al. (2000) 
identified factors associated with poor comprehension by family members of the status of ICU patients. These factors were found to be patient-related, family-related, or physician-related factors. Patient-related factors included being less than 50 years old, being unemployed, being referred from a hematology or oncology ward, being admitted for acute respiratory failure or coma, and having a reasonably favorable prognosis. Family-related factors for poor comprehension were being of the same culture, not being the spouse, and lack of a healthcare professional in the family. Physician-related factors included lack of time spent in the first family meeting (less than 10 minutes) and failure to give the family an information brochure (Azoulay et al., 2000).

Family members of ICU patients also experience high levels of depression and anxiety. In a large, prospective, multicenter study in French ICUs, 637 patients and 920 family members, had symptoms of anxiety (69.1\%) and depression (35.4\%). These symptoms were present in $72.7 \%$ of family members and $84 \%$ of spouses. Factors that contributed to anxiety included: absence of chronic disease in the patient, spouse being female, absence of regular physician and nurse meetings, and absence of a room used strictly for family meetings. Factors that contributed to depression included: patient's age, spouse being female, cultural variations, no waiting room, and perceived contradictions in the information provided by caregivers (Pochard, et al., 2001).

Finally, survivors of the ICU experience high levels of post traumatic stress disorder (PTSD) (Davydow, Gifford, Desai, Needham, \& Bienvenu, 2008). Davydow et al. conducted a systematic review to summarize and critically review data regarding the prevalence of PTSD in ICU survivors, risk factors for post-ICU PTSD, and the impact of post-ICU PTSD on healthrelated quality of life (HRQOL). Clinically significant PTSD symptoms were self-reported in $22 \%(\mathrm{n}=1,104)$ of ICU survivors and clinician-diagnosed PTSD was reported in 19\% $(\mathrm{n}=93)$ 
of ICU survivors. Reliable predictors of post-ICU PTSD included: prior psychopathology, greater use of benzodiazepines, and post-ICU memories of frightening and/or psychotic experiences that occurred while being a patient in the ICU. Consequently, post-ICU PTSD was associated with substantially lower HRQOL (2008).

Impact to WV. Appalachia is a nearly 205,000-square-mile region that follows the backbone of the Appalachian Mountains from southern New York to northern Mississippi (Appalachian Regional Commission, 2011). West Virginia is the only state entirely encompassed by this region. There are about 77 persons per square mile in $\mathrm{WV}$, compared to 124 persons per square mile in the Appalachian region as a whole (Appalachian Regional Commission, 2011). Because of WV's rural mountainous terrain, healthcare is often difficult to access, which contributes to WV's ranking as the unhealthiest state in the country (Milken Institute Chronic Disease Index, 2011). Contributing factors to this dismal ranking lie in the characteristics of the population. Over half of West Virginians are either obese or overweight. Almost $25 \%$ of the population smokes, while only $16.2 \%$ routinely eats the recommended servings of fruits and vegetables daily. Only $32 \%$ of West Virginians obtain the recommended amount of daily physical activity. West Virginians also have increased disparities in income, education, and insurance. Nearly $20 \%$ of West Virginians live in poverty, $16 \%$ have not graduated from high school, and 22\% do not have health insurance (United States Census Bureau, 2011).

In 2012, 2,536 patients in WV received care from a palliative care consult service, which was a 15\% increase in the total number of consults from 2011 (Emmett, Dean, \& Kemper, 2013). The main reasons cited for consultation were goal clarification $(77.7 \%)$, psychological/spiritual support of patient/family (13.7\%), pain and symptom management (5.5\%), and disposition 
(3.2\%). Cancer represented the largest primary diagnosis $(34.5 \%)$ followed by neurological diseases $(14.6 \%)$ for patients that were seen by a palliative care team in WV. A total of 2,300 patients $(91.9 \%)$ had multiple chronic illnesses. Of the 2,536 patients that received palliative services within WV, 401 patients $(16.1 \%)$ went home with hospice, 310 patients $(12.5 \%)$ were discharged home, 232 patients $(9.3 \%)$ went to a nursing home or skilled nursing facility, and 415 patients $(16.7 \%)$ went to a hospice house. Alternatively, a total of 643 hospitalized or nursing home patients $(25.9 \%)$ died in their current setting. Of these 643 patients that died, 390 patients $(63.8 \%)$ died in an acute care bed, 171patients $(28.0 \%)$ died in the ICU, 11 patients $(1.8 \%)$ died with hospice services being utilized in their current setting, and 21 patients $(3.4 \%)$ died in a skilled nursing facility (Emmett, Dean, \& Kemper, 2013).

West Virginians also experience similar barriers in receiving palliative care in the ICU as the rest of the country. In a study by Moss, Demanelis, Murray, \& Jack, (2005), 626 physicians and nurses practicing in 28 hospitals in West Virginia completed surveys regarding the effectiveness of palliative care in the ICU. Results showed that both physicians and nurses agreed on the top three barriers to palliative care in the ICU, which are patients' and families' frequent demands for all possible treatments, inadequate insurance coverage, and lack of health care professional education (Moss, Demanelis, Murray, \& Jack, 2005).

Impact at project medical center. In 2009, there were 90,080 persons living in Monongalia County, WV. Almost $11 \%$ were over the age of 65 , compared with $15 \%$ over the age of 65 in the state of WV (US Census Bureau, 2010). The major city in Monongalia County is Morgantown. It has two hospitals. One is a 189-bed community hospital and the other is a 521-bed academic medical center. Due to the rural nature of WV, both hospitals provide medical services to patients throughout the state. In 2012, 876 patients at the academic medical 
center received palliative care services from an inpatient palliative consult team, comprised of three physicians, two nurse practitioners, a nurse coordinator, a social worker, and a chaplain. For the 872 patients for which data was collected, $85.4 \%(\mathrm{n}=745)$ had multiple chronic illnesses, 267 patients $(32.72 \%)$ had a cancer diagnosis and 194 patients (23.77\%) had a neurological diagnosis. The main reasons for consults at this academic medical center were predominantly goal clarification $(75.32 \%)$, pain and symptom management $(10.33 \%)$, or psychological or spiritual support (13.66\%). Interestingly, pain and symptom management was discussed and recommendations were made $56 \%$ of the time, even though it was not the reason for the initial consult (Emmett, M., Dean, S. \& Kemper, S., 2013).

\section{Problem Statement}

Twenty percent of all Americans die in an ICU or shortly after an ICU stay (Angus et al., 2004). For most of these patients, relentless and distressing symptoms are commonplace (Puntillo et al., 2010). The majority of patients admitted to the medical ICU service at the selected hospital are at high risk of death, making palliative care a top priority in their plan of care. High risk of death in the ICU was defined based upon the literature (Rost et al., 2008;

Norton et. al., 2007) and by local ICU and palliative care experts as meeting at least one of the criterions listed below.

a) ICU admission following a current hospital stay of greater than 10 days

b) Age greater than 80 years in the presence of two or more life-threatening co-morbidities [e.g., end-stage renal disease, severe congestive heart failure (CHF)]

c) Diagnosis of an active stage IV malignancy

d) Status post cardiac arrest

e) Diagnosis of an intracerebral hemorrhage ( $\mathrm{ICH})$ with a functional outcome risk stratification scale (FUNC) score of four or less 
a) End Stage Chronic Obstructive Pulmonary Disease (COPD), lung disease, or CHF Even though palliative care has been recognized as a national priority (IOM, 2001), few patients receive palliative measures on a consistent basis. The implementation of the Communication and Care Bundle for medical ICU patients at high risk of death will standardize the completion of specific palliative measures by specific time frames. There are nine palliative measures in the Communication and Care Bundle (See Appendix C, Table 1). These nine care processes, which are quality measures, are documented in the medical record at key time frames and are listed below:

By ICU Day 1:

(1) Identification of appropriate decision maker

(2) Advance directives

(3) CPR status

(4) Distribution of information leaflet to patient families

(5) Optimal pain assessment

(6) Optimal pain management

By ICU Day 3:

(7) Social work visit

(8) Pastoral care or spiritual support offering

By ICU Day 3:

(9) An interdisciplinary family meeting

\section{Theoretical Framework}

Adoption of the Communication and Care Bundle into the medical ICU is challenging due to the multitude of healthcare providers that work in that setting. Rogers' (2003) diffusion 
of innovation (DOI) was selected for this capstone project because its theoretical base guides the adoption of new innovations and addresses person-specific readiness for change.

\section{Key Elements}

The purpose of the DOI theory is to describe how an "idea perceived as new, spreads via certain communication channels over time among its members in a social system" (Rogers, 2004, p. 13). It is important to differentiate between diffusion, dissemination, implementation, and adoption. Diffusion is a natural process of knowledge-spreading. Dissemination is a planned and active process of knowledge spreading. Implementation is the introduction of an innovation into daily practice. Finally, adoption is the acceptance of the innovation into practice (Achterberg, Schoonhoven, \& Grol, 2008).

There are four main elements of the DOI theory: the innovation, the communication channels, time, and the social system. Innovation is an "idea or practice perceived as new by an individual or other unit of adoption (organization)" (Rogers, 2003, p 12). It does not matter if the idea is truly new. It only matters that the idea is perceived as new. Therefore, whether the innovation is new or old is determined by the individual (Rogers 2003). The perceived qualities of innovation include: relative advantage, compatibility, complexity, trialability, and observability. "Innovations that are perceived by individuals as having relative greater advantage, compatibility, trialability, and observability, and less complexity" (p. 16) are adopted more rapidly than other innovations (Rogers, 2003).

Communication channels are the way in which a message travels from person to person on the basis of a social process. Diffusion itself is a specific type of communication. Diffusion is specifically concerned with the communication exchange of the new idea from an individual to others. More specifically, diffusion occurs between individuals (or whole units of adoption) that 
have experience with the innovation to other individuals (or whole units) that do not have experience with the innovation. This process primarily occurs via informal communication channels heavily influenced by modeling and imitation by potential adopters of their team members who have already adopted the innovation (Rogers, 2003).

The concept of time is also important to understand. According to Rogers (2003), there are three major time dimensions involved in the DOI theory. The first dimension is the time period in which the innovation-decision process occurs. This period encompasses the time in which the individual progresses from "first knowledge of the innovation through its adoption or rejection" (p.20). The second time dimension involves the "innovativeness" (p. 20) of the individual (or whole unit). Innovativeness is defined as the relative "earliness/lateness with which an innovation is adopted" (p. 20) compared to other individuals (or whole units). Finally, the third time dimension is the innovation's rate of adoption into a system, usually defined by the volume of individuals who have adopted the innovation in a given time period.

Looking more closely at the first dimension of time in Rogers' (2003) diffusion of innovation theory, there are five major steps within the innovation-diffusion process. These time-ordered steps include: knowledge, persuasion, decision, implementation, and confirmation. Knowledge is gained as individuals (or whole units) acquire awareness about the existence of the innovation and increase their understanding of how the innovation works. Persuasion occurs as individuals (or whole units) form opinions about the innovation. Decision follows with individuals actively participating in activities that lead to the adoption or rejection of the innovation. Implementation involves putting the new idea to use. Finally, confirmation occurs when individuals (or whole units) seek reinforcement of the decision (Rogers, 2003). In each stage of the innovation-decision process, there are specific tasks or questions to be addressed to 
facilitate the adoption of that stage and the innovation as a whole.

The diffusion process is also very dependent upon the innovativeness of the members of the social system. These members are identified based upon the relative earliness or lateness of their adoption of the innovation. Rogers (2003) classified these individuals into five adopting categories: innovators, early adopters, early majority, late majority, or laggards. Innovators are "active information seekers about new ideas." (p. 22). They are the first to adopt an idea or innovation and do not depend upon others' viewpoints of the innovation. Early adopters are deemed successful users of new ideas, and have a high degree of opinion leadership within a system. Early adopters are the individuals to "check with" (p. 283) for information and advice about the innovation. The category of the early majority is characterized by individuals who adopt the innovation sooner than most and comprise about one third of the system. These individuals interact often within the system, but are not necessarily considered leaders. The late majority also represent about one third of the system, but are usually skeptical of new ieas. The late majority will eventually concede to the pressures to conform to system norms. Finally, laggards are the last individuals in the social system to adopt. They have very little opinion leadership and tend to base their opinions on past experiences. Laggards are most hesitant to adopt and often must be certain the new idea will be successful before they buy into it.

\section{Theory Validity to Proposed Project}

Since the birth of the DOI theory, a large number of studies from different disciplines using this theory have been published. To date, there have been over 5,000 studies on the DOI theory (Rogers, 2004). Those who have used this theory, include not only behavioral health disciplines, but also geography, political science, anthropology, marketing, business management, and public health. 
In healthcare, one of the most recognized applications of the diffusion model was used in the STOP-AIDS program in San Francisco during the AIDS epidemic in the 1980s. The STOPAIDS intervention was extremely successful and used Roger's theory as its basis for implementation. STOP-AIDS assumed that if a critical mass of opinion leaders in the gay community could be reached, that the innovation of using condoms would spread spontaneously to the rest of the population. Following the intervention of the STOP-AIDS intervention, the rate of unprotected anal intercourse dropped from $71 \%$ in 1983 to $27 \%$ in 1987 , and the number of new HIV infections dropped from 8,000 in 1983 to 650 in 1985 (Rogers, 2004).

Due to its wide use by multiple disciplines, the DOI theory is very adaptable to any scientific discipline. Its universality is especially useful for nursing with the professions many varied sub-specialties. In order to make the DOI theory more applicable to nursing, specific nursing-related strategies to promote the adoption of the innovation and the development of more time-sensitive indicators that describe the progression of the diffusion should be utilized. Since most nurses reportedly prefer to learn from other nurses (Thompson, McCaughan, Cullum, Sheldon, \& Raynor, 2001), the DOI theory's use of opinion leaders is aligned with this aspect of nursing culture; thus making it very practical when implementing an innovation in the intensive care unit (ICU)

\section{Theory Application to Current Project}

The World Health Organization's (WHO) (2012) definition of palliative care is aligned with the palliative processes in the Communication and Care Bundle. The WHO asserts that palliative care is:

an approach that improves the quality of life of patients and their families facing the problem associated with life-threatening illness, through the prevention and relief of 
suffering by means of early identification and impeccable assessment and treatment of pain and other problems, physical, psychosocial and spiritual (para. 1).

The DOI theory by Everett Rogers has played a key role in the integration of the Communication and Care Bundle in the ICU. This theory examines how people experience and adopt new ideas or innovations. The DOI theory will, over time, continue to guide nurses to adopt new protocols and guidelines, into their practice. In this study, Roger's theory was utilized to integrate the Communication and Care Bundle within a 18-bed medical and 12-bed surgical ICU of a level one trauma center and university teaching hospital in northern WV. By using this theory and carefully anticipating the diffusion process, most of the Communication and Care Bundle's implementation was managed proactively.

When implementing a practice change such as the integration of the Communication and Care Bundle into daily practices in the ICU, the use of opinion leaders or early adopters in the nurses' and physicians' working social systems served to foster the adoption of the new practice. The early adopters were champions for the guideline implementation and supported the change whole-heartedly. At each stage in the diffusion process, the opinion leaders were ready to address questions that may have hindered the adoption of this evidence-based protocol. In the knowledge acquisition stage, both an online educational program and ICU opinion leaders answered questions such as: What is the Communication and Care Bundle? How does it work? Why does it work? The opinion leaders also ensured that the nurses, physicians, and others in the medical ICU knew what the protocol was and had access to the plethora of data supporting its use.

In the persuasion stage, opinion leaders' knowledge about the advantages and disadvantages of this practice protocol was used to address concerns over outcomes associated 
with the Communication and Care Bundle. They used their influence as unit formal and informal leaders to persuade ICU staff. Unit-wide persuasion affecting all health care disciplines was an important strategy to aid in the adoption of the Communication and Care Bundle.

In the decision stage, the opinion leaders continued to use interpersonal channels of communication to facilitate the adoption of the protocol into daily practice. Since pilot-testing is useful in this stage, only patients admitted to the medical ICU service were considered for inclusion into this project. As the adopted protocol continues to become fully integrated into the daily practice of this sub-population and positive outcome data continues to be collected regarding the intervention's effectiveness, other ICUs will be invited to adopt the protocol as well. Successful implementation of the palliative care intervention with the medical ICU patients as a pilot population will reassure other ICU nurses and healthcare providers the intervention works.

In the implementation stage, nurses and the nursing leadership put the palliative processes of the Communication and Care Bundle to use. Questions addressed in this stage included: Where do I gain access to the guideline? How do I use it? How much time will it take and how will that affect my day to day patient care? If it affects my care negatively, how will I correct this? How will the leadership team of the medical ICU provide the support needed to sustain this change?

In the confirmation stage, it is likely and expected that the nurses, healthcare providers, and organization leaders may question the benefits of the Communication and Care Bundle. The opinion leaders must stand true to their beliefs at this point. Their conviction will serve as reinforcement to the new adopters of the change. The medical and surgical ICUs are currently in this stage. 
The rate of adoption of the clinical protocol is depicted by a graph shaped like an " $\mathrm{S}$ " (see Figure 2). The s-shaped curve depicts the relative speed in which the members of a social system, in this case, the ICU nurses, adopt the new clinical practice. Empiric evidence in over 5,000 research studies since the 1940's all point to the s-shaped curve to predict how fast the innovation will be adopted (Rogers, 2003). Opinion leaders of the ICU were quick to see the value in this project and adopt the innovation early, as is depicted in the time axis of the s-curve. The opinion leaders were followed by early adopters, early majority, and late majority. The last group to adopt the change will be the laggards (Shirley, 2006), and are usually the individuals most resistant to change. It is likely that there are still some laggards that have not adopted the Communication and Care Bundle. If Roger's DOI theory holds, this group will adopt the Communication and Care Bundle slowly over time, as evidence supports its utility.

\section{Literature Review and Synthesis}

Current research regarding both individual and multifaceted strategies for improvement of palliative care in the ICU is abundant. However, the integration of palliative care into the ICU setting via the Communication and Care Bundle is revolutionary for this field. Therefore, the purpose of this literature review is to determine the state of the science regarding what interventions have been effective in improving palliative care in the ICU especially focusing on the nine quality measures highlighted in the Communication and Care Bundle (Appendix C, Table 1).

\section{Methodology}

Search terms restricted eligible studies by using a combination of specific keywords in the methodological search of five scholarly databases. The databases included CINAHL, PubMed, Education Research Complete, and Health Source - Nursing/Academic Edition. The initial 
search using the phrase, Communication and Care Bundle resulted in four studies (Nelson, Mulkerin, Adams, \& Pronovost, 2006; Penrod, Luhrs, Livote, Cortez, \& Kwak, 2011; Penrod et al., 2012; Norton, et al., 2007). To broaden the search, new search terms were implemented and included palliative care and ICU, and were limited to the past ten years, with peer-reviewed journals only. This initial search yielded a total of 372 hits. The titles of all 372 articles were reviewed for relevance. Although many titles were duplicated or were not relevant to this literature review, there were still over 200 relevant titles identified. The search was further narrowed at this point to obtain the most relevant research. This was done by including the aforementioned keywords and the new search term, quality improvement. The search was then further defined by limiting the years searched to 2006-2012, peer-reviewed journals only, adult patient population, and ICU setting. Using these limitations, this search resulted in 33 hits. Results were further excluded if they were not a clinical guideline, systematic review, quantitative study or a qualitative study. After these exclusions, there were 16 relevant studies available for review, which then produced 13 relevant results including those mentioned previously regarding the Communication and Care Bundle. A review of references of the existing literature was then undertaken to ascertain that all relevant articles were obtained. This review led to the identification of 15 additional articles, 3 of which were relevant, thereby producing a total of 2 clinical guidelines and 14 research studies for review.

\section{Critical Appraisal}

The planned search produced a total of 2 clinical guidelines [National Consensus Project (NCP) for Quality Palliative Care, 2013; National Quality Forum's (NQF) Framework and Preferred Practices for Quality Palliative and Hospice Care, 2011], 3 RCTs (Curtis et al., 2011; Lautrette et al., 2007, SUPPORT Investigators, 2006), 1 combination case-control, descriptive, 
qualitative study (O’Mahoney et al., 2010), 9 descriptive studies (Billings et al., 2006; Curtis, 2008; Daly et al., 2010; Lilly et al., 2000; Nelson, Angus et al., 2006; Nelson, Mulkerin et al., 2006; Norton, 2007; Penrod, Luhrs, Livote, Cortez, and Kwak, 2011; Penrod, 2012), and 1 qualitative study (Nelson, 2010). A critical appraisal was conducted on the two clinical guidelines using the AGREE II Instrument (Brouwers, et al., 2010) and on the two RCTs using the appropriate SIGN tool developed by the Scottish Intercollegiate Guideline Network (2007). Eight quantitative studies were individually described and appraised for the internal validity using the "Synthesis Worksheet for Quantitative Evidence" (Larrabee, 2009). One qualitative study was described and appraised using the "Literature Review Worksheet for Qualitative Research Studies" (Larrabee, 2009). This literature is reported by hierarchy of evidence then by earliest publication year first.

\section{Clinical Guidelines}

Because many patients in the ICU are at high risk for death and most have distressing, unrelieved symptoms (Puntillo et al, 2010), improvement of palliative care in the ICU has been identified as a national health priority (IOM, 2001). The Center to Advance Palliative Care (CAPC), reports that improving palliative care in the ICU is acknowledged as a priority by all major organizations representing professionals caring for critically ill patients and their families (2012). Relevant national guidelines for clinical practice include the NCP for Quality Palliative Care (2013) and the NQF's Framework and Preferred Practices for Quality Palliative and Hospice Care (2011). These guidelines have set forth a national standard for palliative care for all clinical settings in which seriously ill patients and their families receive treatment.

The NCP for Quality Palliative Care (2013) provides a framework for the foundation of essential palliative principles and practices. This foundation is sorely needed as palliative care 
programs are growing in response to patient need. These guidelines provide structure for the assessment and treatment of pain and other distressing symptoms, help with patient-centered communications and decision-making, and support in the coordination of care across various care settings. The NCP Clinical Practice Guidelines for Quality Palliative Care (2013) represent consensus among nationally recognized experts, and is based upon current, relevant research. The guidelines also describe core instruction related to clinical palliative care programs and are divided into eight dedicated sections: structure and processes of care, physical aspects of care, psychological and psychiatric aspects of care, social aspects of care, spiritual, religious and existential aspects of care, cultural aspects of care, care of the patient at end of life, and ethical and legal aspects of care.

The NCP for Quality Palliative Care (2013) was appraised for this project, using the AGREE II Instrument (Brouwers, et al., 2010) to assess the quality of the guideline, evaluate the process of the practice guideline development, and the review the quality of reporting of the guideline. Based upon this appraisal, the guideline received a 4 out of 7 or $57 \%$ overall score, which deemed useful and recommended with modifications. The guideline was particularly weak regarding its descriptions of the criteria for selection of evidence, methodology of data collection, strengths and limitations of the evidence, guideline development process, and the process in which health benefits, side effects and risks were considered when formulating the recommendations. Other recommended changes would be to enhance the applicability domain by specifically addressing how the guideline can be put into practice, identifying potential resource implications of applying the recommendations, and including the presentation of monitoring or auditing criteria. Finally, within the editorial independence domain, the guideline failed to address that the views of the functioning body did not influence the content of the guideline and 
competing interests were recorded and addressed. Cumulative scores for each domain are as follows: $89 \%$ for Scope and Purpose; 94\% for Stakeholder Involvement; 52\% for Rigor of Development; 94\% for Clarity of Presentation; 25\% for Applicability; and 0\% for Editorial Independence.

The NQF has recently acknowledged palliative care and hospice care as national priorities for healthcare quality improvement. The high-ranking NQF report, Framework and Preferred Practices for Quality Palliative and Hospice Care (2011), provides a framework and set of NQF-endorsed preferred practices that focus on improving palliative care and hospice care across the IOM's six dimensions of quality, which are: safe, effective, timely, patient-centered, efficient, and equitable. These preferred practices mark a crucial step in the standardization of palliative care and hospice. Every process measure identified in the Communication and Care Bundle has been endorsed by the NQF (2011).

The NQF's Framework and Preferred Practices for Quality Palliative and Hospice Care (2011) was also appraised for this project, using the AGREE II Instrument (Brouwers, et al., 2010), to assess the quality of the guideline, evaluate the process of the practice guideline development, and the review the quality of reporting of the guideline. The guideline was rated a 6 out of 7 or $86 \%$ overall, which deemed it useful and recommended with modifications. These modifications include making the rigor of the guideline more transparent, specifically by discussing the systematic methods that were used to search for evidence and adding descriptions of the criteria for selection of evidence. Other recommended changes would be to enhance the applicability domain by increasing information on how the guideline can be put into practice and further identifying potential resource implications of applying the recommendations. Finally, within the editorial independence domain, the guideline was limited in content addressing how 
the functioning body did not influence the content of the guideline, and competing interests were recorded and addressed. Cumulative scores for each domain are as follows: $100 \%$ for Scope and Purpose; 100\% for Stakeholder Involvement; 69\% for Rigor of Development; 100\% for Clarity of Presentation; 75\% for Applicability; and 83\% for Editorial Independence.

\section{Research Studies}

Studies supporting palliative strategies. Only one of three RCTs supported the use of various palliative care strategies to promote palliative care integration into the ICU. A singleblind RCT evaluated a format consisting of a proactive end-of-life conference and a brochure to lessen the effects of bereavement (Lautrette et al., 2007). The study occurred in 22 ICUs in France from May 2005 to October 2005. Fifteen ICUs were in teaching hospitals and seven in general hospitals. Randomization was performed centrally in blocks of six, stratified according to the ICU, with group assignments sent in sealed envelopes to the study centers. A total of 126 family members were initially enrolled to complete a 90-day follow-up phone interview after their ICU experiences. A total of 108 families (86\%) completed the interview process. Fifty-six families received the new intervention format compared to 52 families who received the customary end-of-life conference. The patient's characteristics did not differ significantly between the two study groups. Families in the intervention group $(n=56)$ were given a brochure on bereavement and participated in an end-of-life conference with predetermined guidelines. On day 90 , the 56 participants in the intervention group who responded to the telephone interview had a significantly lower median Impact of Event (IES) score than the 52 participants in the control group (27 vs. $39, \mathrm{P}=0.02)$ and a lower prevalence of PTSD-related symptoms (45\% vs. $69 \%, \mathrm{P}=0.01$ ). The median Hospital and Anxiety and Depression Scale (HADS) score was also lower in the intervention group (11 vs. 17 in the control group; $\mathrm{P}=0.004)$. Symptoms of 
both anxiety and depression were less prevalent in the intervention group versus the control group (anxiety $45 \%$ vs. $67 \%$; $=0.02$; depression $29 \%$ vs. $56 \% ; p=0.003$ ). The major strength of this study is its randomization across multiple French ICUs. Limitations of the study include some differences between the hospitals at baseline. Three hospitals already had been using protocols for delivering bad news to patients and families and five of the hospitals already had open visiting hours, which has been shown to be reflective of a more patient and family centered ICU. Also, $11 \%$ of the subjects dropped out prior to the intervention and intention to treat analysis was poorly addressed. Thus, there was no accountancy of why there this attrition occurred.

One combination case control, descriptive, qualitative study (O’Mahoney et al., 2010) was successful in providing a culturally competent model of care for critically ill patients at the end-of-life. This study used a convenience sample of 157 consecutive patients cared by an integrated ICU and palliative care team within a multi-site medical center in the northeast US. Retrospective chart review data was abstracted from the medical record with a standardized chart abstraction instrument, and family focus groups were conducted. The focus group interviews were conducted with a sample of family members and ICU nurses to rate the quality of end-oflife care in the ICU by using the Quality of Dying and Death in the ICU instrument (ICUQODD) for patients who died in an ICU bed during the study period. A separate case-control study was conducted comparing the length of hospital stay for persons who died in the ICU during the final six months of the project. Comparisons were made between groups that did receive a palliative care consultation and groups that did not receive a palliative care consultation. Pharmacoeconomic data were evaluated for 22 patients who died with a palliative care consultation and 43 patients who died without a palliative care consultation. The goal of the case-control study was 
to determine if there was an association with an increase in the use of pain medications or alterations in the use of potentially non-beneficial life-prolonging treatments in persons dying in the intervention ICU. After the intervention of 157 consecutive palliative care consultations, $62.4 \%$ of patients' and families' identified significant spiritual needs. Education on the death process was provided to $85 \%$ of families by the project team. Twenty-nine percent of patients were weaned from ventilators. Vasopressor support was stopped for almost $16 \%$ of patients. Artificial nutrition was discontinued $15.3 \%$ of the time. Six percent of patients stopped dialysis and $2.5 \%$ discontinued artificial hydration. Recommendations regarding pain management were made $51 \%$ of the time and symptom management were made $52 \%$ of the time. Finally, DNR orders and hospice referrals increased from $33 \%$ prior to consultation to $83.4 \%$ after the intervention. The length of time from admission to a palliative care consult at the intervention ICU was 2.8 days versus 15.5 days $(\mathrm{p}=0.0184)$. Palliative care consultation did not impact median survival times (12 days at the control site and 13.5 days at the intervention site). A major strength of this study was its comprehensive methodology. Limitations to this study include its potential lack of generalizability due to it being conducted at a single medical facility, although different sites were used for the pharmaco-economic data collection. Another limitation was that the response rates were low for both the qualitative interviews and the case control study. Additionally, the evaluation team and respondents were non-blinded; therefore, respondents may have been biased.

Seven descriptive studies (Billings et al., 2006; Lilly et al., 2000; Nelson, Angus et al., 2006; Nelson, Mulkerin et al., 2006; Norton et al., 2007; Penrod, 2011; Penrod, 2012) showed positive results when examining the relationships between specific palliative interventions and improved process and outcomes measures associated with palliative care in the ICU. Penrod et 
al. (2011) implemented the time triggered, evidence-based interventions of the Communication and Care bundle in 5 Veteran Administration (VA) hospital ICUs. Researchers recruited 415 patients (176 during baseline and 239 post-intervention), who were mostly men $(\mathrm{n}=406$ or $97.8 \%$ ), with an average age of 70 years. Fifty percent of the sample was Caucasian, with $38.6 \%$ having a diagnosis of pulmonary disease. Critical care and palliative care providers educated ICU nurse teams to improve care through chart audits, performance feedback, use of improvement tools, staff education, and monthly team meetings. The greatest improvement was in the proportion of patients for whom an offer of social work support was documented (22\% to $60 \%, \mathrm{p}<0.001$ ). Identification of a medical decision maker also improved from $40 \%$ to $52 \%$ (p $<0.02$ ) and offering spiritual support to patients and/or families by day three increased from $35 \%$ to $45 \%$ ( $\mathrm{P}<0.05)$. Documentation of interdisciplinary family meetings by day five increased from $13 \%$ to $20 \%$ ( $\mathrm{P}<0.04)$. Investigation of CPR status improved from $81 \%$ to $87 \%$, but the change was not significant $(\mathrm{P}<0.08)$. Finally, determination of advance directive status went down from $85 \%$ to $75 \%$ ( $\mathrm{P}<0.003)$. One possible explanation offered by researchers for this result is that the ICU nurses may have focused more on the assignment of a medical decision maker and CPR status, versus the clarification of advance directives status. A strength of this study rests in it data collection methodology. Chart review data was collected only by a paid research assistant, thus inter-rater reliability concerning consistency in data collection was not an issue. One limitation to the study was its lack of generalizability. The sample was mostly men, veterans, and had a common diagnosis of pulmonary disease, and may not translate to other populations of patients.

Like the study by Penrod et al. (2011), a later descriptive study by Penrod and colleagues, (2012) measured the percent of ICU patients with lengths of stay of five or more days that 
received the palliative care processes identified in the Communication and Care Bundle by the appropriate day. The study used a convenience sample consisting of adult patients with an ICU length of stay of five or more days in five different ICUs (one surgical, two medical in academic medical centers, and two medical in community hospitals). The average age of patients was 62.7 years. Across all sites, the prevalence of performance of specific care processes ranged from $8 \%$ for distribution of a family information leaflet to $80 \%$ for appropriate pain management. Pain measures were highest for assessment (76\%) and management (80\%) whereas performance on other day one measures (identification of medical decision-maker, investigation for advance directive, determination of CPR status, distribution of family information leaflet) were lower $(8 \%-43 \%)$. Most of the patients spoke English and were Caucasian, and had family members that visited daily. Approximately one-third of patients received an offer of spiritual support or social work support by day three, and only $20 \%$ of the patients had an interdisciplinary family meeting by day five. A strength of the study was the sample size, [518 (94.9\%) patients and 336 $(83.6 \%)$ family members].

Although one study is (Lilly et al., 2000) twelve years old; it is still worth review. The study was a pre/post, descriptive study, in which the authors compared usual care with a proactive, multidisciplinary method of communication, which averaged 90 minutes in length. The multidisciplinary meetings occurred with $270(68 \%)$ of 396 of the patients in the intensive communication group or their families within 72 hours of ICU admission, including $99 \%$ of the targeted group of 273 patients. A greater percentage of intensive communication meetings were held in the post-intervention period than in the pre-intervention period [273 of 396 (69\%) vs. 58 of $134(43 \%), P<0.001]$. The authors also showed that intensive communication significantly reduced the median length of stay from 4 days to 3 days, $(p=0.01)$, without increasing mortality. 
In fact, there was a decrease in unadjusted overall mortality $(\mathrm{p}=0.02)$ and a trend toward reduced mortality during the ICU stay $(\mathrm{p}=0.06)$. A strength of this study was its relatively large sample size ( $\mathrm{n}=530$ adult medical patients). One limitation of this study is that the intensive communication intervention lasted an average of 90 minutes. Other sites may not be able to make such a time commitment, due to staffing limitations or availability of meeting rooms. The study also did not use randomization, which would have strengthened its design. Even with the limitations, the results were significant.

Another descriptive study by Nelson, Angus et al. (2006) used a self-administered mail survey to ask participants about barriers to end-of-life care, the perceived benefit of strategies to improve end-of-life care, and availability of these strategies. Completed surveys were received from 590 of 1,205 $(49.0 \%)$ ICU directors, with nurse directors $(\mathrm{n}=406)$ and physician directors $(n=184)$ comprising $68.8 \%$ and $31.2 \%$ of the sample respectively. More than $80 \%$ of respondents rated all 14 palliative strategies as being helpful. One of the strengths of this study was that the surveys were randomly mailed to ICU directors from 468 of 600 or $78 \%$ of ICUs in 48 states, plus the District of Columbia. The fact that nurses had a response rate that was double that of physicians may cause bias and difficulty in generalizing the results.

Nelson, Mulkerin et al. (2006) used a pilot implementation project to evaluate the feasibility and baseline ICU performance of the nine care processes of the Communication and Care Bundle. The authors found documentation of a family meeting for 34 out of 85 (40\%) of patients who were in these ICUs for at least five days. Medical records also reflected that an information leaflet was distributed to 38 out of 88 (43\%) of families, and 33 families (37.5\%) received an offer of spiritual support by day three in the ICU. ICUs included in the pilot test fared best on pain assessment with pain scores documented for 521 out of $600(87 \%)$ in four- 
hour nursing intervals, and records showed that pain was alleviated during 411 out of 482 (85\%) intervals examined. The high rating for pain management may be due to these same hospitals already being involved in an initiative to enhance pain assessment and management. Like the studies by Penrod et al. (2011) and Penrod et al. (2012), this study's strength rests on the fact that the interventions are individually supported by the strongest evidence in the existing literature. The study would have been much stronger if post-intervention data was evaluated prior to publication. This study was limited by a small sample size of 5 medical-surgical units, 4 four medical units, 3 surgical units, and 4 mixed units from a total of 10 separate hospitals $(n=94$ patients).

Norton et al. (2007) used a prospective, pre/post design to examine the effect of proactive palliative care consultation on length of stay for high-risk patients in a 17-bed MICU. Patients (n =191) were identified at high risk of dying upon admission to the MICU as meeting at one of the following criteria: a) intensive care admission following a current hospital stay of greater than 10 days; b) age greater than eighty years in the presence of two or more life-threatening comorbidities; c) diagnosis of an active stage IV malignancy; d) status post cardiac arrest; or e) diagnosis of an intracerebral hemorrhage requiring mechanical ventilation. During an initial four-month "usual care" phase, consultations for patients $(n=65)$ occurred when a MICU physician made a referral through the standard referral mechanisms that existed before the study. Following this four-month "usual care" baseline period was a seven-month intervention phase, in which all patients who met one of the inclusion criteria received either a basic or complete palliative care consultation. During the usual care phase, the average number of days from MICU admission to complete palliative care consultation was 14. During the intervention phase, all 126 patients received a basic palliative care consultation within 1.73 days and 31 (25\%) went 
on to receive a complete palliative care consultation within 4.9 days from MICU admission. There was no significant difference $(p<.6128)$ between mortality rates in the usual care $[\mathrm{n}=25$ of $65(38.5 \%)]$ and the intervention groups [n= 46 of $126(36.5 \%)]$. There was no difference in the hospital length of stay for the usual care group: 41.40 days compared with 35.8 days for the proactive PC intervention group $(\mathrm{p}<.5011)$. There also was no significant difference $(\mathrm{p}<$ 0.0510) between the length of stay of the hospital decedents (excluding MICU decedents) $(\mathrm{n}=$ 9.17 days in the intervention group and $n=16.09$ in the usual care group. However, the decedents in the intervention group had significantly $(\mathrm{p}<.0001)$ shorter MICU lengths of stay (8.96 vs. 16.28). One limitation of the study was that the patients receiving only the basic palliative care consultation were not followed throughout their hospitalization after their MICU stay. The authors conclude that this may have likely diluted any potential effect of the intervention on length of stay for the period between MICU admission and hospital discharge. One of the strengths of this study is that the usual care and intervention groups were similar in respect to age, gender, race, screening criteria, or discharge disposition, thus making it more likely that the intervention of a proactive palliative care consultation played a role in decreasing ICU length of stay for patients identified to be at high-risk of dying.

Billings et al. (2006) used a descriptive exploratory design to review the key interventions and general findings of a three-year project that was designed so palliative care and intensive care physicians would share their expertise and develop projects that promote end-oflife care in a MICU setting. Subjects were nurses and physicians located at an urban, tertiary care, academic hospital. The MICU was a closed unit with about 12 intensivists trained in pulmonary and critical care and responsible for all patients. The main interventions reported as being helpful included: nursing and physician collaboration, palliative care nurse champions, 
staff education, palliative care specialist on rounds for modeling and teaching key behaviors, promotion of family meetings, open visiting hours, a poster to help preventing patient anonymity, and staff support. The major limitation to this study is that the authors reported on preliminary results only, thus evaluation of the intervention's effectiveness could not be ascertained without post-intervention data. Although the preliminary results are promising, postintervention data is a stronger source of evidence than the authors' observations.

Finally, a qualitative study conducted by Nelson (2010) helped to identify how patients and families define high-quality intensive care. Forty-eight subjects (15 patients, 33 family members) in nine focus groups across three ICU sites completed focus groups led by a single physician facilitator. Domains and processes of care that emerged included: timely, clear, compassionate communication by clinicians; clinical decision making focused on patients' preferences, goals, and values; patient care maintained in comfort, dignity, and personhood and family care with open access and proximity to patients; interdisciplinary support in the ICU, and bereavement care for families of patients who died. One strength of this study lies in its description of the analytic process. The authors' used a domain analysis strategy in the interpretation of their findings. This analysis used three investigators to independently code meeting transcripts, and then achieve consensus on themes. The authors then used software to sort passages according to codes and examine relationships among the codes and coded passages. This process validated the coders' initial framework. Another strength of this study is that the focus groups used open-ended, exploratory questions to gain insight into the participants' point of view. A primary limitation of this study is that its design limits generalizability. Furthermore, subjects were excluded if they lived more than an hour's drive to the interview site, or were not 
proficient in the English language. These two exclusions may have biased the sample to urban, American-born participants.

Studies with mixed results. Curtis (2008) conducted a descriptive study to evaluate the effectiveness of a multifaceted quality improvement intervention to improve palliative care in the ICU. Like the study by Curtis et al. (2010), this study's intervention consisted of academic detailing, which involves face-to-face education of healthcare providers of medical evidence which has been shown to improve patient care and reduce health care costs (O'Brien, Rogers, \& Jamtvedt, 2007). Other interventions included clinician education, local champions, feedback to clinicians, and system support. The nurse ICU Quality of Death and Dying (ICUQODD) questionnaire showed significant improvement (pre-intervention, 63.1; post-intervention, 67.1; $\mathrm{p}$ $<0.01$ ), and there was a significant reduction in patient ICU days before death (pre-intervention, 7.2; post-intervention, 5.8; $\mathrm{p}<0.01$ ). The family ICUQODD questionnaire showed a trend toward improvement (pre-intervention, 62.3; post-intervention, 67.1) as well, but the findings were not statistically significant $(p=0.09)$. Family satisfaction [via the Family Satisfaction in the ICU (FS-ICU) scale] increased, from a pre-intervention score of 80.8 (SD, 16.2) to a postintervention score of 83.0 (SD, 16.5), but not to the level of statistical significance $(\mathrm{p}=0.14)$. One possible reason for the lack of significance from the families' ratings is the low response rate. Only $55 \%$ of families $(n=225)$ completed the family ICUQODD. Although the responding families were not statistically different from each other, they were statistically different from non-responding families. Families without questionnaires were more likely to be Hispanic, and had loved ones with shorter lengths of stay. Due to these limitations, interpretation of the families' results may not be generalizable to more diverse populations. A 
strength of this study was that the nurses response rate for completion of the ICUQODD was $89 \%(n=523$ out of 590$)$.

Nonsupportive studies for palliative care strategies. An unblinded RCT by Curtis et al. (2010) did not support the use of a five-component intervention to promote palliative care in the ICUs. The study's intervention targeted clinicians with the following components: clinician education, local champions, academic detailing, clinician feedback of quality data, and system supports, and was delivered by experts in palliative care from outside of the institution. The primary outcome, family ICUQODD, showed no change with the intervention $(P=0.33)$. There was also no change in family satisfaction via the FS-ICU $(\mathrm{P}=0.66)$ or nurse's ratings of patients quality of death and dying via the ICUQODD $(\mathrm{P}=0.81)$. There was a non-significant increase in ICU days before death after the intervention (hazard ratio $=0.9 ; \mathrm{P}=0.07$ ), which may actually represent prolongation of dying. Finally, among patients undergoing withdrawal of mechanical ventilation, there was no change in time from admission to ventilator withdrawal (hazard ratio $=$ $1.0 ; \mathrm{P}=0.81)$. The strengths of the study were that it was a cluster randomized trial, which is the gold standard of research design. The treatment groups' differences were controlled for by weighted analysis through regression models using instruments that have a history of being valid and reliable. The study also had several limitations. Despite, the large initial enrollment numbers $(n=2,318)$, potential response bias should be considered due to the poor response rates of nurses $(27-39 \%)$ and families $(43-44 \%)$. Also, the number of hospital sites was only 12 , and the hospitals themselves were also innately different. There was one university-affiliated hospital, three non-university teaching hospitals, and eight non-teaching hospitals. The most plausible explanation as to why the intervention was unsuccessful is that since it was administered by experts from outside the facility, communication between the patients, families, 
and the outside experts was less effective than it would have been if delivered by the healthcare team directly. Also, there was less collaboration between the experts and healthcare team and no required family meetings, which both interventions have been shown to be extremely effective in limiting unwanted and ineffective aggressive treatment at end-of-life.

The SUPPORT study was a two-year prospective, observational study (phase I) with 4,301 patients followed by a 2 two-year controlled clinical trial (phase II) with 4,804 patients and their physicians. The participants were randomized by specialty group to the intervention group $(n=2,652)$ or the control group $(n=2,152)$. The study was conducted in five teaching hospitals in the US, and had a total sample size of 9,105 adults hospitalized with at least one of nine lifethreatening diagnoses, and an overall six-month mortality rate of 47\% (SUPPORT Investigators, 1996). During phase I, the pre-intervention phase, inadequacies in communication, occurrence of aggressive treatment, and the characteristics of hospital death were monitored. Astoundingly, only $47 \%$ of physicians knew when their patients preferred to avoid CPR; $46 \%$ of DNR orders were written within two days of death; $38 \%$ of patients who died spent at least 10 days in the ICU; and of $50 \%$ of conscious patients who died in the hospital, family members reported moderate to severe pain at least $50 \%$ of the time (SUPPORT Investigators, 1995). Following the pre-assessment phase, intervention consisted of the physician group receiving communications regarding six-month prognosis estimates of their patients, outcomes of CPR, and predicted functional disability of their patients at two months. A nurse trained in palliative care also initiated contacts with the patient, family, physician, and hospital staff to determine patient and family preferences, discuss patient outcomes, highlight pain control, discuss end of life options, and facilitate family meetings. Sadly, during phase II of the study, patients did not experience an increase in patient-physician communication. Furthermore, patients' written DNR orders, CPR 
preferences, length of stay in the ICU (receiving mechanical ventilation, or comatose before death), level of reported pain, and use of hospital resources did not change (SUPPORT Investigators, 1996).

Daly et al. (2010) also did not show a positive correlation between a structured, intensive communication system (ICS) and length of stay among long-stay ICU patients. The control group (usual care) consisted of 135 patients and their families. Subjects were enrolled from five ICUs at two academic medical centers, a surgical ICU (SICU), MICU, and neuroscience ICU at a university-affiliated, not-for-profit medical center, and a MICU and SICU at a universityaffiliated public medical center in the same city. The authors then implemented the intervention and enrolled 354 patients and family members. The ICS included a family meeting within five days of admission and weekly thereafter. Each meeting discussed goals of care, a medical update, values and preferences, treatment plan, and milestones for judging effectiveness of treatments. The authors found no significant reduction in LOS among the sickest patients. Limitations of this study were that the ICUs were inherently different, and the odds of receiving a tracheostomy were significantly related to the admitting unit (medical ICU less than a nonmedical ICU). Possible reasons that the ICS was not effective maybe related to the length of time of the ICS itself, lasting only 30 minutes for the intervention group. Additionally, the ICS was often replaced by a quicker bedside communication due to the inability to congregate family and medical team members simultaneously. Finally, there was not an intervention to train healthcare providers on how to conduct an effective family meeting. Given these limitations, this study's results should be interpreted with caution.

\section{Synthesis}


Based upon the conclusions of the critical analysis, the body of evidence to determine which palliative strategies resulted in improved palliative care in the ICU was similar in nine included studies (Billings et al., 2006; Lautrette et al., 2007; Lilly et al., 2000; Nelson, Angus et al., 2006; Nelson, Mulkerin et al., 2006; Nelson et al., 2010; Norton, et al. (2007); O’Mahoney et al., 2010; Penrod, et al., 2011; Penrod, 2012;). One study produced mixed results (Curtis, 2008). Three studies did not endorse any specific findings to improve palliative care in the adult ICU (Curtis et al., 2011; Daley et al., 2010; SUPPORT Investigators, 1996).

The majority of strategies cited as increasing palliative process measures and improving patient or family centered outcomes recommended collaboration among all members of the healthcare team, especially between physicians and nurses (Billings et al., 2006; Lautrette et al., 2007; Lilly et al., 2000; Nelson, Angus et al., 2006; Nelson, Mulkerin et al., 2006; Nelson, 2010; Norton, et al., 2007; O’Mahoney et al., 2010; Penrod, 2011). The use of timely, patient centered, interdisciplinary family meetings was also cited to improve palliative care in the ICU (Billings et al., 2006; Lautrette et al., 2007; Lilly et al., 2000; Nelson, Angus, et al., 2006; Nelson, Mulkerin et al., 2006; Nelson, 2010; Norton et al., 2007; Penrod, 2011). Other strategies that were found to increase the palliative care process in the ICU were system or staff support measures, for instance ancillary services such as pastoral care and social services (Billings et al., 2006; Nelson, Angus, et al., 2006; Nelson, Mulkerin, et al., 2006; Nelson, 2010; Norton, et al., 2007; O’Mahoney et al., 2010; Penrod, 2011) and frequent symptom assessment and management (Billings et al., 2006; Nelson, Angus, et al., 2006; Nelson, Mulkerin, et al., 2006; Norton et al., 2007; O’Mahoney et al., 2010; Penrod, 2011; Pronovost et al., 2012). Fostering a patientcentered culture was also recognized as being integral to enhancing palliative care in the ICU (Billings et al., 2006; O’Mahoney et al., 2010). Less cited, but still relevant studies that 
supported successful strategies to promote palliative care in the ICU included the identification of the patient's medical decision maker (Billings et al., 2006; Penrod, 2011) and formalization and documentation of advance directives early in the patient's hospitalization (Billings et al., 2010; O’Mahoney et al., 2010; Penrod, 2011). These two processes often led to increased DNR orders (Billings et al., 2010; O’Mahoney et al., 2010; Penrod, 2011) as patients had more interactions in formulation of goals of care. Brochures for families on bereavement (Billings et al., 2006; Lautrette et al., 2007; Nelson, Angus et al., 2006; Nelson, 2010) and an introduction to palliative care brochure (Nelson, Mulkerin, et al., 2006) also showed positive correlations with effective palliative care in the ICU. Education of effective end-of- life communication and other palliative issues (Billings et al., 2006; Daly et al., 2010) and open visiting hours (Billings et al., 2006; Nelson, 2010; O’Mahoney et al., 2010) were also found to be effective in integrating palliative care into the ICU.

Some single descriptive exploratory studies produced support for certain strategies to improve ICU palliative care. Billings et al. (2006) were the only authors to show the benefit of palliative care nurse champions and the use of a "Get to Know Me" poster. Another study asserted that scheduling nurses to care for the same patient each day (Nelson, Angus, 2006) led to better patient outcomes and an increase in core palliative processes. Nelson et al. (2006) reported that supervised training and role modeling of the healthcare team from palliative care specialists, increased contact with palliative and ethics specialists, the use of formalized symptom assessment tools, and increasing resources for the accommodation of diversity led to better patient outcomes and increased palliative processes. Finally, Norton et al. (2007) showed that proactive palliative care consultation could result in reduced MICU length of stays while not affecting mortality for patients at high risk of dying 
Three studies (Curtis et al., 2008; Curtis et al., 2011; Daley et al., 2010) reported no significant differences in palliative care processes after a combined intervention of staff education, audit and feedback, academic detailing, use of palliative care champions, and staff support. However, the study by Curtis et al. (2008) provided evidence of an increase in nurse related scores on ICUQODD. In this descriptive study, there was no significant improvement in family satisfaction scores with either the FS-ICU scale or the ICUQODD scale; however there was an increase in the nurses' satisfaction scores on the ICUQODD.

Like the later RCT study by Curtis et al. (2011), the intervention was an interdisciplinary multifaceted intervention of clinician education, local champions, academic detailing, clinician feedback of quality data, and system supports. Again, the intervention did not consist of a family meeting, which multiple studies have shown to be positively correlated with improved palliative care. The authors proposed that improving family ratings may require interventions that have more direct contact with family members. Also, according to Daley et al. (2010), the use of an intensive communication system did not improve ICU length of stay.

There is a plausible explanation why the RCT by Curtis et al. (2011) did not impact palliative care processes. First and foremost, the intervention did not consist of a family meeting. This literature review has shown that most successful interventions to improve palliative care in the ICU almost always include a family meeting. This study was also implemented in the ICU by experts outside the institution, negating the positive effects that collaboration can have in the fruition of a palliative care initiative.

There are gaps in the evidence. While many studies have reported how specific strategies have improved domains of palliative care, few have identified which combination of strategies is most effective. The three studies that reviewed palliative processes of the Communication and 
Care Bundle (Nelson et al., 2006; Penrod et al., 2011; Penrod et al., 2012) are the exception.

These studies consistently show an increase in palliative care processes after the implementation of the bundle strategies. However, the number of studies is currently limited to just three; thus, more research is needed to strengthen this connection.

Overall, the strength of the body of evidence comprised of two clinical guidelines, three RCTs, one case-control study, eight descriptive studies, and one qualitative study is sufficiently strong to draw conclusions. The nine care process of the Communication and Care Bundle shows promise in consistently increasing the domains of palliative care in the ICU. In conclusion, it seems clear that the interventions used to introduce palliative strategies into the ICU should be collaborative, adapted to the individual ICU's culture, involve the multidisciplinary team, and be patient and family centered.

\section{Congruence of the Organization's Strategic Plan to Project}

Many federal and professional agencies work to promote the delivery of palliative care, including the Joint Commission on Accreditation of Hospitals and Organizations (JCAHO) (JCAHO, 2008) and the American Nurses Credentialing Center (ANCC) Magnet recognition program (ANCC, 2012). JCAHO standards that are related to the scope of palliative care include: the patient's right to and need for effective communication, pain assessment and management, coordination of care, and comfort and dignity. Palliative care processes are also aligned with the ANCC's Magnet Recognition program by the following forces: Force 6: Quality of Care; Force 7: Quality Improvement; Force 8: Consultation and Resources; Force 13: Interdisciplinary Relationships (ANCC, 2012).

The organization in which this performance improvement project was implemented is a large, 531-bed tertiary care and academic medical center, located in northern West Virginia. It 
has been has been nationally recognized as a level one trauma center and has received Magnet recognition for nursing. Embodying the philosophy that "the patient is first in everything we do," the mission of this academic medical center is "to improve the health of West Virginians and all we serve through excellence in patient care, research, and education." (WVU Healthcare, 2011, para 1). This is the essence of the institution's self-reported reason for existing. This institution encourages its employees to live this mission every day. Organizational specific performance improvement initiatives are currently in place at the academic medical center in which the project will be implemented. The particular initiatives that are closely tied to palliative care domains within this organization include improving pain assessment and management and reducing length of stay.

\section{Project Description}

\section{The IPAL Project}

To operationalize the national mandates for improved quality palliative care, the CAPC developed the Improving Palliative Care (IPAL) Project. This initiative was designed to provide a central site for "sharing expertise, evidence, tools, and resources essential to the integration and improvement of palliative care in specific health care settings" (para 1). Located within the IPAL website are numerous quality improvement tools for improving palliative care. These tools were developed by colleagues at several institutions across the country. They are accessible to healthcare providers who can access tools continuously, free of charge. Together with co-sponsorship from the National Institutes of Health (NIH), CAPC later launched the IPAL-ICU Project (CAPC, 2012, para 1). The IPAL-ICU Project is a new initiative that focuses specifically on improving palliative care in the ICU. 
The Communication and Care Bundle. One tool highlighted on the IPAL-ICU website is the Communication and Care Bundle developed by the Voluntary Hospital Association (VHA), Inc. The VHA is a cooperative network of more than $25 \%$ of US not-for-profit, community-based hospitals (Nelson, Mulkerin, Adams, \& Pronovost, 2006). The Communication and Care Bundle began as part of the VHA's "Transformation of the ICU" performance improvement initiative. This initiative involved interdisciplinary critical care teams from over 80 hospitals across the country, who implemented "bundles" of quality measures in a variety of critical care practice areas (Nelson, Mulkerin, Adams, \& Pronovost, 2006). The measures in this bundle are linked to domains of quality established by the NCP for Quality Palliative Care (2009) and to relevant practices set forth in the NQF's Framework and Preferred Practices for Quality Palliative and Hospice Care (2011). Currently, the National Quality Measures Clearinghouse website of the Agency for Healthcare Research and Quality (2012) has listed the Communication and Care Bundle as part of its databank for quality measurement, improvement, and reporting.

What is unique about this initiative is the concept of "bundling" palliative care. A bundle is "a small set of evidence-based interventions for a defined patient segment/population and care setting, that when implemented together, will result in significantly better outcomes than when implemented individually" (Resar, Griffin, Haraden, \& Nolan, 2012, p. 2). To date there have been 5 bundles that have shown significant improvement for patient care by the Institute for Healthcare Improvement (IHI): the Central Line Bundle, the Ventilator Bundle, 2 Severe Sepsis Bundles, and Perinatal Care Bundles. There are other bundles in development, but data related to their efficacy is still somewhat limited. 
There are nine palliative measures in the Communication and Care Bundle. These nine care processes, which are quality measures, are documented in the medical record at key time frames (see Appendix C, Table 1). On ICU day one (the day after ICU admission) the following measures are initiated: identification of appropriate decision maker, advance directives, CPR status, distribution of information leaflet to patient families, and optimal assessment and management of pain. Identification of an appropriate decision maker included the formalization of either a healthcare surrogate or a MPOA. Advance directive include any of the following: healthcare surrogate, MPOA, physician scope of orders and treatment (POST form), DNR card, or a living will. CPR status was defined as either a full code or do not resuscitate. The distribution of an information leaflet to patient families (Appendix D), was created prior to the implementation of this performance improvement project, but has not been consistently supplied to families in the past. The leaflet describes the role of the Supportive Care team within the academic medical center, how to reach the team. For this performance improvement project, the information leaflet was included in the patient admission packet to the ICU and placed in the waiting room outside the MICU and SICU. Optimal assessment of pain is defined as documentation of a pain score (either numeric or behavioral) at minimum every four hours. Optimal management of pain is defined by a numeric pain score of 5 or less, and a behavioral pain score of two or less. On or before ICU day three, documentation a social work consult occurs and pastoral or spiritual support is offered.

Finally, on or before ICU day five, an interdisciplinary family meeting should occur. The original Communication and Care Bundle requires a family meeting for all ICU patients, since all ICU patients are seriously ill. In the setting of this performance improvement project, palliative care attending physicians and medical ICU attending physicians agreed to target 
patients at high risk for death for the structured family meeting. Interdisciplinary is defined as being a minimum of two different healthcare disciplines, such as medicine, nursing, social work, or pastoral care. This academic medical center's Supportive Care team is multidisciplinary and consists of physicians, nurse practitioners, a nurse coordinator, a social worker and a chaplain. Other disciplines committed to the care of palliative patients in this medical center include respiratory care, physical therapy and occupational therapy. Each member of the multidisciplinary team attends weekly interdisciplinary team meetings to review current patient case loads and plan for the appropriate interdisciplinary care of their patients.

Again, for this performance improvement project, patients at high risk of death in the ICU was defined by the literature (Rost et al., 2008; Norton, et. al., 2007) and by local ICU and palliative care experts as meeting at least one of the criteria listed below.

a) ICU admission following a current hospital stay of greater than ten days

b) Age greater than 80 years in the presence of two or more life-threatening co-morbidities [e.g., end-stage renal disease, $\mathrm{CHF}$ ]

c) Diagnosis of an active stage IV malignancy

d) Status post cardiac arrest

e) Diagnosis of an ICH with a FUNC score of four or less

f) End-stage COPD, lung disease, or CHF

\section{Primary Research Question}

Does a nurse-led intervention to promote the adoption of the Communication and Care Bundle in the medical and surgical ICUs increase the documentation of the nine quality measures of the Bundle?

\section{Secondary Research Question}


Does the adoption of the Communication and Care Bundle in a medical and surgical ICU decrease ICU length of stay without increasing mortality for adult, medical ICU patients at high risk of death?

\section{Project Objectives}

There are nine palliative measures in the Communication and Care Bundle. These nine care processes, which are quality measures, should be documented in the medical record at key time frames for patients meeting inclusion criteria. Specific objectives were evaluated based upon the following research questions and are outlined as below:

\section{By Day 1:}

1. Does the use of the Communication and Care Bundle for adult, medical ICU patients at high risk of death increase the percentage of patients with identification of appropriate decision maker documented in the electronic medical record (EMR)?

2. Does the use of the Communication and Care Bundle for adult, medical ICU patients at high risk of death increase the percentage of patients with advance directives documented in the EMR?

3. Does the use of the Communication and Care Bundle for adult, medical ICU patients at high risk of death increase the percentage of patients with DNR versus full code documented in the EMR?

4. Does the use of the Communication and Care Bundle for adult, medical ICU patients at high risk of death increase the percentage of patients with documented distribution of information leaflet to patients and/or families in EMR? 
5. Does the use of the Communication and Care Bundle for adult, medical ICU patients at high risk of death increase the regular pain assessment (percentage of 4 hour intervals with documentation of pain assessment) in EMR?

6. Does the use of the Communication and Care Bundle for adult, medical ICU patients at high risk of death increase optimal pain management as recorded in EMR (percentage of 4 hour intervals in which pain rating is less than five on a numeric pain scale, less than two on the PAIN-AD scale, or less than two on the Critical Care Pain Observation Tool (CPOT) scale?

\section{By Day 3:}

7. Does the use of the Communication and Care Bundle for adult, medical ICU patients at high risk of death increase the percentage of patients who had a social work consult documented in the EMR?

8. Does the use of the Communication and Care Bundle for adult, medical ICU patients at high risk of death increase the percentage of patients who had a spiritual support consult offered documented in the EMR?

\section{By Day 5:}

9. Does the use of the Communication and Care Bundle for adult, medical ICU patients at high risk of death increase the percentage of patients who had an interdisciplinary family meeting documented in the EMR?

\section{Two other research questions address patient outcomes.}

10. Does the use of the Communication and Care Bundle for adult, medical ICU patients at high risk of death decrease their ICU length of stay? 
11. Does the use of the Communication and Care Bundle for adult, medical ICU patients at high risk of death affect patient mortality? (If the patient dies while in the ICU or is discharged from ICU and dies within 24 hours, this will be considered as an ICU death).

\section{Project Design}

\section{Performance Improvement Project Intervention Plan}

This capstone project used a descriptive, pre/post design to determine the effectiveness of a nurse-led performance improvement project. It employed multifaceted interventions to ensure successful implementation of the Communication and Care Bundle. The multifaceted intervention consisted of academic detailing of nurse and physician leaders, educational sessions of bedside nurses, meetings with nursing staff, strategically placed posters, and an implementation of a bedside nurse checklist.

\section{Stakeholder Support}

In order to make the implementation of the intervention successful, stakeholder motivation for change was immediately addressed. Addressing key factors that support motivation to change behavior assisted in the successful integration of the Communication and Care Bundle in the ICU. Key stakeholders in this performance improvement project were physician and nursing leaders, bedside nurses, social workers, pastoral care, and the patients and families.

Physician stakeholders. Physician stakeholder includes both the palliative care physicians and the medical ICU physicians. Early physician involvement reinforces centrality of their role in patient care. Demonstration of how the initiative is aligned with patient-centered goals and increased efficiency is another important motivator for changing behavior among physicians (Welch, 2010). Increased presence of the change agent, knowledge of reimbursement 
issues related to the subject (Manaker, Krier-Morrow, \& Pohlig, 2010), and exposure of the physicians to the evidence (Welch, 2010) are integral for a successful change strategy with this group of stakeholders. Multiple meetings with the Medical Director and Section Chief of the ICUs and the Medical Director for Supportive Care facilitated physician support in for this performance improvement project. A project brief was developed and used to facilitate understanding among these stakeholders (see Appendix E). Finally, a follow-up email was constructed and sent to the Medical Director and Section Chief of the ICU at his request to share with his house staff.

Nurse stakeholders. Approval from nursing leadership was required to implement the Communication and Care Bundle in the medical ICU. Impetus to change for this group of stakeholder rested in showing how the project was aligned with key nursing quality indicators. Key measures that the facility was addressing at the time of project implementation included Magnet re-certification, JCAHO accreditation, and hospital-specific performance improvement measures of improved pain assessment and management and decreased length of stay. The project brief (see Appendix E) was also utilized with this group of stakeholders as well.

Bedside nurses played a key role in the integration of the Communication and Care Bundle in the medical ICU. Nurses often do not recognize their value as a communicator and a change agent for their patients (Chiplasky, 2013). A multifaceted strategy was implemented for the nurses focusing on their role in the implementation of the Communication and Care Bundle. This strategy consisted of an interactive computer based learning (CBL) module (see Appendix F), displaying 3 colorful informative posters (see Appendix G) in strategic locations, implementation of a bedside checklist (see Appendix H), and several meetings with the nursing staff. 
Ancillary stakeholders. Although led by nurses, the implementation of the Communication and Care Bundle required a multidisciplinary effort. Other key stakeholders included pastoral care and social services. These groups of healthcare providers played a significant role in their consultative expertise for the targeted patient population. These providers were well informed regarding their importance in the success of this project. Informative meetings with the leadership of these departments were held prior to the implementation of the Communication and Care Bundle to gain this group's support.

Communication to the pastoral care team was initiated by the Director of Pastoral Care regarding the project and to the care management team (consisting of nurse case managers and social workers) via staff meetings. Follow-up informative emails were constructed and sent to the leadership of both services to facilitate communication and provide updates.

Patient and family as stakeholders. The Communication and Care Bundle was designed specifically to improve the quality of end of life for the patients and families in the ICU. The patients and families associated with this study were expected to gain direct benefit from the performance improvement project, though no direct interaction occurred between patients, families, and researchers. Evidence exists that through the use of the specific interventions in the Communication and Care Bundle, patients and families will experience more patient-centered care, alignment of care with patient values and goals, and experience less depression, anxiety, and post traumatic stress disorder (Lautrette's et al.'s, 2007).

\section{Patient Population}

This performance improvement project took place in a combined three-unit medical ICU, with six beds each (total 18) and a 12-bed surgical ICU in a 521-bed academic medical center in Northern West Virginia. The maximum nurse to patient ratio is one to two. Types of patients 
include trauma, neurosurgery, vascular, general surgery, general medicine, stroke, pulmonary, metabolic disease and renal disease. The specific patient population that was targeted for this change project was adult patients who were admitted to the MICU service located on either the SICU or MICU physical units and identified as being at high risk of death. The nurses of both the MICU and SICU worked interchangeably on both units.

Nationally recognized definitions of stage IV malignancy, end-stage heart failure, and end-stage COPD and lung disease were used to maintain consistency in patient inclusion. Stage IV malignancy was defined by the National Cancer Institute (NCI) (2013) as any cancer that has spread to distant tissues or organs. Because accurate prognostication in heart failure, COPD and lung disease is still commonly disputed, end-stage heart failure was defined using guidance from End of Life Palliative Education Resource Center (EPERC) Fast Facts \#143 (Reisfield \& Wilson, 2005), and end-stage COPD and lung disease was defined using guidance from EPERC Fast Facts \#141 (Childers, Arnold, and Curtis, 2005), for hospitalized COPD patients. Additionally, the FUNC score, a valid tool for predicting patients' functional independence 90 days after ICH (Rost et al., 2008), was used in this project to identify patients with poor prognoses.

\section{Sampling Plan}

Power analysis was used to calculate the minimum sample size required to determine an effect of the given sample size. A power analysis was performed prior to data collection for this performance improvement project. It was determined that a sample size of 55 was needed both before and after the intervention to obtain a power of 0.80 . Although there are no formal standards for power, most nurse researchers consider a power 0.80 as the standard for adequacy. With a power of 0.80 , it can be reasonably concluded that a significant difference in the pre- 
intervention data and the post-intervention data was caused by the intervention, and not random chance.

A convenience sample of 55 patients that met inclusion criteria was audited both before and after the intervention via the EMR. Pre-intervention data collection included chart reviews from a six month time period of December 1, 2012 to May 1, 2013 prior to the intervention. Post-intervention data collection began six weeks after the intervention, to allow for adoption of the Communication and Care Bundle into practice. The post-intervention data collection occurred between August 15, 2013 and September 25, 2013.

\section{Data Collection}

Pre-Intervention data collection. All adult medical ICU patients that were either in the physical MICU or SICU, from the period of December 1, 2013 through May 1, 2013, that met inclusion criteria (Appendix I) were identified through the decision support team within the academic medical center. These patients were then screened for inclusion into the project. If the patient met inclusion criteria, their chart was audited using a chart abstraction tool (Appendix L). The chart abstraction tool was utilized to obtain information regarding the documentation frequencies of the nine palliative process of the Communication and Care Bundle (Appendix C, Table 1), and to determine length of stay and patient mortality. If the patient died within 24 hours of ICU discharge, it was considered as an ICU death.

Waiting period. After the one month (July 1, 2013 through July 31, 2013) of baseline academic detailing, education at nursing staff meetings, completion of an online computer-based learning module (CBL) (Appendix F), and implementation of three colorful reminder posters (Appendix G) and a nurses' bedside checklist (Appendix H), there was a six-week waiting period to allow the diffusion of the education into practice. 
Post-intervention data collection. Beginning August 15, 2013, data collection for patients in the post-intervention group began. During the pre-intervention chart review and the initial phases of the post-intervention chart review, the realization was made that many high-risk patients with shorter ICU lengths of stay were also benefiting from the Communication and Care Bundle. In order to capture this data, the requirement for an ICU length of stay of five or more days was removed from the post-intervention inclusion criteria. New inclusion criteria were developed for the post-intervention phase (see Appendix J) and approved by the Institutional Review Board (IRB) (see Appendix K). This performance improvement project's primary research question was to assess if a nurse-led intervention increased quality measure of the Communication and Care Bundle at key time frames. The nurses were instructed to use the inclusion checklist (Appendix H) to identify patients who could benefit most from the planned use of the Communication and Care Bundle. Nurses were not to wait five days to intervene with these palliative processes. The ICU staff nurses played no role in determining who was included in this project. If the patient met the criteria for high risk of death, regardless of their length of stay, nurses were educated to be proactive, implement, and advocate for the palliative processes of the Communication and Care Bundle on or before the key time frames of day one, three and five. Ideally, similar patient groups would have been required to draw conclusions about the patient outcomes of length of stay and mortality.

Multifaceted interventions to increase project adoption. The multifaceted interventions to promote adoption of the Communication and Care Bundle in the medical ICU included the following strategies:

1. Academic detailing with both nursing and physician leadership was utilized at baseline and at periodic intervals to address concerns. 
2. Nurses were educated regarding the nature of the project, their role in the success of the project, and the potential benefits of the project via an online CBL (Appendix F) and unitbased, nursing staff meetings.

3. Three colorful posters (Appendix G, Figure 4), placed in strategic locations determined by nursing leadership were displayed as a reminder of the nine processes in the Communication and Care Bundle.

4. Nurses also had access to a bedside checklist (see Appendix H), which detailed the key processes and time frames of the Communication and Care Bundle.

\section{Resources}

The overall total project costs were $\$ 23,192.46$ (Appendix M). This included a total funding for personnel salaries of $\$ 22,990$ (Appendix M, Table 1). These costs were deferred since the fees were integrated into existing job responsibilities of all persons involved. Nonhuman costs totaled $\$ 202.46$ (Appendix M, Table 1). Supplies utilized for the implementation of this project were provided by the investigators.

While the project did not incur any direct costs to the ICU, the additional time was required by nurses to consult the Communication and Care Bundle checklist and to document palliative care had the potential to impact ICU operations. It was expected that there would be some increase in the numbers of palliative care consultations or family meetings, which was acceptable to both the physician leadership of the medical ICU service and the Supportive Care team. Nurses needed time to attend in-services, complete the CBL (Appendix F), and to implement the Communication and Care Bundle for the appropriate patients. These indirect costs to the ICU are part of the mission and vision of the institution and were supported administratively from the nursing leadership of the medical and surgical ICUs. 
One particular ICU resource expected to be used more frequently was the designated family conference room just outside the MICU and one just inside the SICU. These rooms are used solely to conduct family meetings by physicians and nurse practitioners, and are often used by pastoral care for supportive interventions for families. The potential for increased use of these rooms was not expected to impact operations of the MICU or SICU. Since the Communication and Care Bundle involved many care processes that already routinely occur, the complexity of the implementation of this protocol was deemed relatively low.

\section{Evidence of Support}

A letter of support was obtained from the academic medical center prior to project implementation (see Appendix N). The letter was crafted according to nursing services policy requirements for research proposal (WVU Healthcare, 2011). The nursing division of this academic medical center fully supports the conduct and utilization of clinically oriented research and staff participation in research activities is encouraged. This project was approved by an ad hoc team comprised of Nursing Research Council members, led by the Director of Nursing Administration. After approval of this project by the hospital, the protocol was submitted to the IRB and approved as an expedited protocol on May 1, 2013 (see Appendix O).

After approval by the IRB, the Director of Nursing Administration was contacted prior to the initiation of the study with documents supporting the IRB's approval. Periodic updates on the progress of the study were provided to the Director of Nursing Administration, and were reviewed by the Nursing Research Council. A written copy of the results will be provided to the Director of Nursing Administration and shared with the Nursing Research Council.

\section{Evaluation}

Specific process measures and patient outcomes were evaluated as follows: 
1. The percentage of patients with an identification of appropriate decision maker documented in the EMR by day one was evaluated using Chi Square.

2. The percentage of patients with advance directives documented in the EMR by day one was evaluated using Chi Square.

3. The percentage of patients with documented distribution of information leaflet to patients and/or families documented in the EMR by day one was evaluated using Chi Square.

4. The percentage of four-hour interval documentation of pain assessment documented in the EMR by day one was evaluated an independent t-test.

5. The percentage of four-hour intervals in which pain rating is less than five on a numeric pain scale, less than two on the Pain -AD scale, or less than two on the CPOT for patients was evaluated using an independent t-test.

6. The percentage of patients or families who had contact with a member of the Care Management team documented in the EMR by day three was evaluated using Chi Square.

7. The percentage of patients who had a spiritual support consult offered and was documented in the EMR by day three was evaluated using Chi Square.

8. The percentage of patients who had an interdisciplinary family meeting documented in the EMR by day five was evaluated using Chi Square

9. ICU length of stay was evaluated using and independent t-test.

10. ICU mortality was evaluated using Chi Square.

\section{Results}

\section{Quality Measures}

The Communication and Care Bundle's quality measures were reviewed as the percentage of the actual times the quality measure was documented according to its specific time 
frame. These time frames were as follows: at day one (the day after ICU admission)

(identification of appropriate decision maker, advance directive status, CPR status, and receipt of Supportive Care Patient Information Leaflet), at ICU day three (social work visit and spiritual support offering), and at ICU day five (interdisciplinary family meeting).

Day 1. The frequencies and percentages of the quality measures for both the preintervention phase and post-intervention phase for day one are described in Table 1, Appendix P. These quality measures were evaluated using Chi Square. The patient's MPOA or surrogate was documented in the EMR 22 times (40\%) in the pre-intervention period and 39 times (70.9\%) in the post-intervention period, which was a significant difference $(\mathrm{p}<0.001)$. Presence of an advance directive was documented in the EMR 22 times (40\%) in the pre-intervention period and 39 times $(70 \%)$ in the post-intervention period, which was also significantly different $(\mathrm{p}<$ 0.001). Resuscitation status was documented in the EMR 55 times $(100 \%)$ in both in the preintervention and post-intervention periods. Full code status was preferred by patients and families 54 times $(98.2 \%)$ in the pre-intervention time period versus 47 times $(85.5 \%)$ in the post-intervention time period. This was a significant change in resuscitation preference $(\mathrm{p}<$ 0.015). Finally, distribution of the patient leaflet was documented zero times in both the preintervention and post-intervention time periods.

Regular pain assessment and optimal pain management documented by day one in both the pre-intervention and post-intervention time periods are listed in Table 2, Appendix P. Both of these quality measures were evaluated using independent t-tests. Regular pain assessment was documented $84.3 \%$ of the time in the pre-intervention time period and $86 \%$ of the time in the post-intervention time period, which did not represent a significant change $[\mathrm{t}=(-) 0.472 ; \mathrm{p}=$ 0.158)]. Surprisingly, optimal pain management was documented $86.2 \%$ of the time in the pre- 
intervention time period and $74.4 \%$ of the time in the post-intervention time period, which was significantly less $(\mathrm{t}=2.350 ; \mathrm{p}<0.01)$ in the post-intervention time period.

Day 3. The frequencies and percentages of the quality measures for both the preintervention phase and post-intervention phase for day three, and was evaluated using Chi Square and are displayed in Table 3, Appendix P. Care Management visits were documented in the EMR 24 times $(43.6 \%)$ in the pre-intervention time period and 31 times $(56.4 \%)$ in the postintervention time period; a statistically significant change $(\mathrm{p}=0.023)$. Spiritual support was offered and documented in the EMR 12 times $(21.8 \%)$ in the pre-intervention time period and 19 times $(34.5 \%)$ in the post-intervention time period, but failed to show a significant difference (p $=0.119)$.

Day 5. The frequencies and percentages of the quality measure for both the preintervention phase and post-intervention phase for day five are depicted in Table 4, Appendix P. Interdisciplinary family meetings were documented 20 times $(36.4 \%)$ in the pre-intervention time period versus 22 times $(32.7 \%)$ in the post-intervention time period. Chi Square was used to determine if there was a significant difference between the numbers of interdisciplinary meetings documented on or before day five in both the pre-intervention and post-intervention time periods. This analysis did not reveal a significant change $(\mathrm{p}=0.471)$ in documented meeting frequencies.

\section{Patient Outcomes}

Because the multifaceted intervention aimed at increasing documentation of the nine quality measures of the Communication and Care Bundle was only successful in three out of nine measures, then it is not appropriate to evaluate ICU length of stay and mortality as it is dependent upon the successful implementation of the Communication and Care Bundle. Even so, 
patient outcomes of ICU length of stay and patient mortality were measured both during the preintervention period and post-intervention period. An independent T-test was used to evaluate the data, with results posted in Table 5, Appendix P. The patients' ICU length of stay was an average of 10.69 days during the pre-intervention time period and an average of 4.89 days in the post-intervention time period. This change represents a significant difference $(\mathrm{p}<0.001)$ in length of stay between the pre-intervention and post-intervention time periods.

Patient mortality was evaluated using Chi Square, and results are displayed in Table P8. A total of 26 out of $55(47.3 \%)$ patients died either during or after an ICU discharge in the preintervention time period. Of these patients, twelve (21.8\%) were considered ICU deaths, while $14(25.5 \%)$ died greater than 24 hours after the ICU discharge. A total of 17 patients $(30.9 \%)$ died in the post-intervention time period. Ten of these patients $(18.2 \%)$ were considered ICU deaths, and seven (12.7\%) died greater than 24 hours after ICU discharge. There was no significant difference $(\mathrm{p}=0.155)$ in mortality between the pre-intervention and post-intervention time periods.

\section{Patient Population}

Patient inclusion criteria differed in the pre-intervention phase and post-intervention phase of this study. Patients with an ICU length of stay of at least five days were included in the pre-intervention inclusion criteria, but not the post-intervention inclusion criteria. Table 7 , Appendix P delineates patient inclusion criteria by frequency and percentage in both the preintervention and post-intervention time periods using Chi Square analysis. The pre-intervention and post-intervention groups were similar with one exception. There was a significantly $(\mathrm{p}=$ 0.022) larger number of patients with end-stage COPD, end-stage lung disease, or end-stage CHF in the pre-intervention group $(n=36,65.5 \%)$ versus the post-intervention time period $(\mathrm{n}=$ 
$24,43.6 \%)$. Otherwise, there were no significant differences in frequencies of specific inclusion criteria in the pre-intervention versus the post intervention groups.

\section{Nurse Demographics}

Table 8, Appendix $\mathrm{P}$ summarized the demographics of the nursing population of the MICU and SICU. Out of 120 nurses, 90 (75\%) of the MICU and SICU nurses completed the online educational module. The nursing population of the MICU and SICU was notably young, (56.7\% were between the ages of 20 to 30 ), baccalaureate prepared (77.8\%), with limited time of employment at the organization (64.4\% had been employed less than five years). Most (61.1\%) had less than five years of nursing experience and slightly more $(63.3 \%)$ had less than five years of ICU nursing experience. Most nurses (73.3\%) did not hold any specialty certification; while only $16.7 \%$ had achieved their Critical Care Nursing Certification (CCRN). After the multifaceted educational intervention, a combined $87.7 \%$ reported that they had either "yes, most definitely" (33.3\%) or "yes, mostly" (54.4\%) gained new knowledge.

Finally, Table 9, Appendix P provides insight into nurses perspectives related to palliative care in the ICU. When asked what the biggest obstacle for providing palliative care was in the ICU, most nurses $(71 \%)$ cited communication problems. Lack of communication between the healthcare team was cited $37.8 \%$ of the time. Lack of communication with families was cited $33.3 \%$ of the time. Knowledge was only identified $6.7 \%$ and time to care for patients as $14.4 \%$. Eighty percent of the nurses reported the biggest area for improvement for palliative care in the ICU was to further develop communication among patients, families, and the healthcare team. Improvement related to symptom management during and after extubation was listed $12.2 \%$ of the time. Finally, spiritual and psychosocial support was recognized only $7.8 \%$ of the time as the biggest area for palliative care improvement in the ICUs. 


\section{Discussion}

This performance improvement project measured the effectiveness of nurses advocating for and coordinating the implementation and documentation of the quality measures of the Communication and Care Bundle for the sickest of patients in the ICU. After a multifaceted intervention to improve nurses' knowledge regarding the Communication and Care Bundle, there were successes. The adoption of the Communication and Care Bundle by nurses has been shown to increase documentation three out of nine quality measures in the MICU and SICU for adult medical ICU patients at high risk of death. There was a significant improvement in the documentation of MPOA or healthcare surrogate $(\mathrm{p}<0.001)$, advance directives $(\mathrm{p}<0.001)$, and care management contacts $(\mathrm{p}<0.023)$ after a multifaceted intervention to integrate quality measures into the care of high-risk of death adult, patients in the MICU or SICU.

DNR preferences were significantly increased $(\mathrm{p}=0.015)$ after the implementation of this performance improvement project. Because the patient groups were different, care must be taken in interpreting the reason for the significant increase in DNR orders from the preintervention phase to the post-intervention phase. It is expected that with longer lengths of stays and more patients with end-stage COPD/lung disease/CHF, there would be more DNR orders in the pre-intervention group than the post-intervention group. This was not the case. Patients with end-stage COPD/lung disease/CHF was significantly less $(\mathrm{p}<0.022)$ in the post-intervention phase (decreased from $65.5 \%$ to $43.6 \%$ ). One explanation for the increase in DNR orders in the post-intervention phase is that there were doubled in the number of patients over the age of 80 years with two or more life-threatening co-morbidities in the post-intervention groups versus the pre-intervention group ( 8 versus 16 respectively). Although, this increase was not significant, it did approach significance $(\mathrm{p}<0.065)$. Another explanation is that patients may have been 
making more informed choices in the post-intervention phase due to increased knowledge and advocacy for palliative care from the nurses.

Optimal pain management significantly decreased $(\mathrm{p}<0.01)$ after the implementation of this performance improvement project, which is counterintuitive. The major reason cited for the decrease in optimal pain management was the implementation of a new pain assessment tool, the CPOT, which specifically assesses pain in intubated, ICU patients after the pre-intervention data collection period ended. It is believed that the presence of pain was recognized more accurately since the implementation of this tool; thus leading to the increased documentation of higher pain values associated with the CPOT. Arbour, Gelinas \& Mauchad (2011) showed an increased frequency of pain assessments and an increase in identification of pain episodes postimplementation of the CPOT. Vázquez, Pardavila, Lucia, Aguado, Margall, \& Asiain (2011) observed ICU patient's behavior using the CPOT during the painful procedures and concluded that the CPOT allowed professionals to better objectify pain in critically-ill patients with verbal communication difficulties. Vázquez et al.'s, (2011) study also highlighted the importance of nurses' to administer of additional analgesia before a painful procedures. It is possible that optimal pain management did not actually decrease, but rather that nurses were better able to objectify patients' pain.

There were quality measures that were not affected by the implementation of the Communication and Care Bundle. These included documentation of the patient information leaflet and regular pain assessment by day one, documentation of spiritual care offered by day three, and documentation of an interdisciplinary care meeting by day five. The documentation of a patient leaflet being given to the patient or family was zero for both the pre-intervention and post-intervention groups. Discussions with ICU nurse leaders revealed that the leaflet had been 
integrated into the admission packets that all patients receive upon admission to the ICU. It is possible that patients received the leaflets in the post-intervention phase, but that it was not documented.

Regular pain assessment increased from $84 \%$ to $86 \%$, but was not significant $(\mathrm{p}=0.158)$. Still, $86 \%$ is a high percentage of assessing pain regularly. It should be noted that a new pain assessment tool, the CPOT, was integrated into normal procedures of the ICU during the waiting period starting on July 1, 2013. As with all new policies, consistent incorporation into practice is sometimes slow at first. It is possible that the integration of the new pain tool blunted the true ability for nurses to more consistently assess pain every four hours per ICU policy. If the Communication and Care Bundle was implemented after this new tool was already established, perhaps the increase in regular pain assessment would have reached significance.

Spiritual care was offered to patients in the pre-intervention group $21.8 \%$ versus $34.5 \%$ of the time in the post-intervention group. These results may have been increased to significance, had the post-intervention group had a longer length of stay. Because the postintervention group had significantly $(\mathrm{p}<0.01)$ shorter lengths of stay $(10.69$ days versus 4.69 days) it is very likely that time constraints may have prevented pastoral care offerings on day three for many that were discharged or died prior to day three.

Interdisciplinary family meetings were held $36.4 \%$ of the time in the pre-intervention period and $32.7 \%$ of the time in the post intervention period. These results may have been different had the inclusion criteria of five or more days in the ICU not been removed from the post-intervention inclusion criteria. Because the post-intervention group had significantly $(\mathrm{p}<$ 0.01) shorter lengths of stay (10.69 days versus 4.69 days) it is very likely that time constraints may have prevented many family meetings. 
The data also showed a significant difference in ICU length of stay $(\mathrm{p}<0.01)$ but not mortality in the pre-intervention and post-intervention groups. Care should be taken when correlating this decrease to the implementation of the Communication and Care Bundle. The pre-intervention group had an additional criterion of a minimum length of stay of at least five days. The pre-intervention group also had a significantly larger number of patients with endstage COPD, lung disease, or CHF ( $\mathrm{p}<0.022)$ compared to the post-intervention group. These factors alone may have skewed the data.

Mortality was not affected by the implementation of the Communication and Care Bundle. There was no significant difference $(\mathrm{p}=0.155)$ in mortality between the preintervention group and the post-intervention group. This is a critical finding; especially in light of the significantly shortened ICU length of stay in the post-intervention group (10.69 days in the pre-intervention group versus 4.69 days in the post-intervention group). Often patients are deferred palliative consults or goal setting discussions because clinicians erroneously believe that these conversations will hasten death. Additionally, palliative care measures are sometimes thought of as secondary treatments in the ICU. Cure versus care has been a long lasting debate in ICU circles. This finding supports the simultaneous use of palliative processes with aggressive therapy.

\section{Limitations}

There were limitations regarding the implementation of this performance improvement project. One of the major limitations was that the inclusion criteria were changed in the postintervention time period in order to capture patients with shorter lengths of stay who were benefiting from the implementation of the Communication and Care Bundle. Subsequently, patients were found to be different in the pre-intervention and post-intervention periods, in 
regards to COPD/lung disease/CHF diagnosis and length of stay. There were significantly fewer ( $p<0.022)$ patients with COPD/lung disease/CHF in the post-intervention group, (decreased from $65.5 \%$ to $43.6 \%$ ). These patients often have long, complicated ICU admissions, and require longer hospitalizations. This difference in patient groups in the pre-intervention and postintervention phases may have contributed to the significantly $(\mathrm{p}<0.01)$ longer length of stay in the pre-intervention phase (10.69 days versus 4.69 days). Additionally, only three of the nine quality measures showed significant improvements, therefore, the Communication and Care Bundle implementation was only partially successful, and could not have fully impacted length of stay even if the groups were similar. For these two reasons, the results regarding ICU length of stay should be interpreted with caution.

Another limitation of the study is that advance directives definition incorporated MPOA and healthcare surrogate during the data collection period. This caused a blurring of these two data sets. In this study, advance directives included MPOA, healthcare surrogate, DNR card, POST forms, and living wills. DNR cards and POST forms are actually medical orders, not advance directives by definition. Advance directives are completed by the patient and are limited in WV to a living will, a medical power of attorney, or a combined living will and medical power of attorney. The advance directives that were documented on day one for this performance improvement project were commonly a combined living will/MPOA. This is the most likely reason that the numbers of MPOA/healthcare surrogates and advance directives were exactly the same in the pre and post-intervention periods. Future studies should define advance directives prior to implementation and differentiate advance directives from medical orders.

A limitation to the project's implementation was that between the times of the preintervention data collection and post-intervention data collection, the ICUs adopted a new pain 
scale, the CPOT. This scale is considered valid and reliable (Gélinas, Fillion, Puntillo, Viens, \& Fortier, 2006), recommended for the intubated critical care population (Arbour \& Gélinas, 2011) and is thought to have led the ICU nurses in this project to conduct more accurate assessment of pain. While identifying patients in pain is the first step in pain management, it is not the only step. Another explanation for the significant decrease in optimal pain management scores $(\mathrm{p}<$ 0.01) may be that there was little education or tools offered to the ICU nurses to effectively manage a now more visible increase in patient pain levels.

\section{Conclusions}

There are national guidelines and sufficient research to support the need for improvement of palliative care for all patients. To improve the quality of palliative care in the ICU, implementation of the Communication and Care Bundle within this project's framework has added to the evidence base. Currently, research is limited in offering ways to increase adoption of the Communication and Care Bundle into ICUs. Additionally, implementing a multifaceted intervention to change clinician behavior can be challenging. Gaining organizational stakeholder support for project implementation has been shown to facilitate the adoption of the Communication and Care Bundle into practice. While only partially successful, this performance improvement project adds to the evidence of nurse-led interventions to promote the adoption of palliative care practices in the ICU.

\section{Additional Findings and Recommendations}

During both the pre-intervention and post-intervention chart reviews, advance directives were not scanned into the EMR until the day of discharge from the hospital or the day of death. For patients that were still hospitalized, it was necessary to search care management, nursing, and physician notes to determine the exact date that the advance directive was implemented. On 
a few occasions, in the post-intervention data collection period, direct visualization of the advance directive on the paper chart was required. Although documentation of the advance directive was stated in the patient summary section of the EMR, actual visualization (scanned copy at discharge or paper copy on bedside chart) of the advance directive was required to determine the exact date of implementation. Because the advance directives were not scanned until the day of discharge or death, the healthcare team may have not known the patient's wishes unless they also reviewed the paper chart for further details. Therefore, it is recommended that the ICU adopts a policy that advance directives are scanned on the first day of ICU admission, or as soon as they become available.

Patients with end-stage COPD, lung disease, or CHF often require longer hospitalizations and may have survived other ICU admissions in the past. Their length of stay is also often considerably longer, than those with other more acute illnesses. These differences may have contributed to the significantly $(\mathrm{p}<0.01)$ longer length of stay in the pre-intervention phase of this performance improvement project. Future research is needed to determine if patients' code status is correlated with surviving past ICU admissions; thereby impacting end of life preferences.

Patients defined as being at high risk of death were limited to the pre-intervention and post-intervention inclusion criteria. It was noted that during the chart reviews, that other patients with terminal diagnoses or events may have been missed for inclusion into this study. Patients with hematopoietic cancers are not classified as stage IV typically. This diagnosis is often limited to solid tumors. Also, patients with severe dementia (FAST score greater than 7A) were not recognized as being at the end-stage of their disease process. Patients with progressive neurological disease, particularly amyotrophic lateral sclerosis (ALS) were not captured as being 
at high-risk of death. Finally, morbidly obese patients with hypoventilation syndrome and hypoxic respiratory failure were not captured unless they also had an underlying disease process identified by the inclusion criteria.

Additionally, when evaluating whether the patient met inclusion criteria, it was noted that several key criteria were difficult to find. Often patients with end-stage disease, a stage IV malignancy, or an ICH with a FUNC score of four or less were not clearly documented as such. Definitions of these diagnoses from the NCI (2013), EPERC Fast Facts \#143 (Reisfield \& Wilson, 2005), EPERC Fast Facts \#141 (Childers, Arnold, and Curtis, 2005), and manual calculation of the FUNC score (Rost et al., 2008) were required to ascertain if the patient was at high risk of death. Because these diagnoses were not easily labeled as end-stage or severe, recognition by the healthcare team of these patients' prognoses may have not been apparent. This may have increased the likelihood of aggressive care and decreased the frequencies of goal clarification within the first five days of ICU admission. Because there are numerous disease states that lead to an ICU admission at end of life, better recognition of patients with life-limiting illness should be adopted.

It is recommended that an interdisciplinary team examines a new, electronic method for capturing end-stage diagnoses, so that it is readily visible in the patient's EMR. Adding the "surprise question" to the delivery of care may be useful in identifying all patients at high risk of death, especially ICU patients. If the physician can say that he or she "would not be surprised if the patient died within the next year," then a palliative consult or goal clarification is warranted. The surprise question has been used for patients with various life-limiting illnesses to assist in recognizing these patients who are appropriate for palliative care (Cohen, Ruthazer, Moss, \& Germain, 2010; Moss et al., 2008; Moss et al, 2010; Pattison \& Romer, 2001) and has been 
found to be an important prognostic indicator in identifying patients with less than one year to live. It is recommended that further discussion in the ICUs reflect upon making life-limiting illness more recognizable at a glance, so patient care and goals can be better aligned.

Further exploration is needed to ascertain reasons why the documentation of the distribution of a patient leaflet by day one, regular pain assessment by day one, spiritual support offered by day three, and the occurrence of an interdisciplinary meeting by day five for patients at high risk of death did not increase. Recommendations include future conversations among the supportive care and ICU interdisciplinary team. Careful review of potential contributing factors should be thoroughly explored. Future research using the same pre-intervention data should be compared with new post-intervention data, incorporating inclusion criteria of at least five days, as in the pre-intervention period.

Finally, inquiry is needed to further define the nurses' perspectives related to obstacles for palliative care in the ICU. Most nurses (71\%) cited communication problems as the biggest obstacle for palliative care in the ICU and $80 \%$ of the nurses reported the biggest area for improvement for palliative care in the ICU was to further develop communication among patients, families, and the healthcare team. Therefore, it is recommended that an investigation of these concerns is jointly explored between the supportive care and ICU nursing and physician teams.

\section{Summary and Implications}

Improvement of palliative care within ICUs in the US and WV is challenging. There were two goals associated with this capstone project. The first goal was to evaluate if a nurse-led intervention to adopt the Communication and Care Bundle increased the implementation of quality measures in the MICU and SICU for adult medical ICU patients high risk of death. 
Documentation of MPOA or surrogate, advance directives, and care management contact all significantly improved after a multifaceted intervention to integrate quality palliative care measures into this patient population. DNR preferences significantly increased, while optimal pain management significantly decreased after this intervention. This decrease was most likely attributable to better pain assessment. Implications for this finding include further education for the ICU staff in treating pain measured via observational pain scales.

The second goal of this project was to evaluate if the Communication and Care Bundle decreased length of stay for adult medical ICU patients at high risk of death in the MICU and SICU without increasing mortality. Length of stay was significantly reduced without affecting mortality. Because of inclusion criteria changes during this project, and only three of the nine quality measures were fully adopted, it cannot be assumed that the Communication and Care Bundle was responsible for the reduction in length of stay. It is important to note that mortality was not affected, even though length of stay in the post-intervention period was significantly shorter $(\mathrm{p}<0.01)$. Implications for this finding support the use of palliative process with aggressive care in the ICU.

Other impacts include identifying ways to mobilize the multidisciplinary team to realizing their role in improving both patient and family quality of life. Crafting a new medical ICU policy regarding key processes for integration upon admission to the ICU and key interventions for the sickest of ICU patients is crucial in enabling early identification of patients at greatest need for palliative services. Further research is needed to determine the best ways to promote palliative care in the ICU and to increase recognition of patients with life-limiting illness, and severe pain. 
Appendix A

Figure 1: Palliative Model of Care

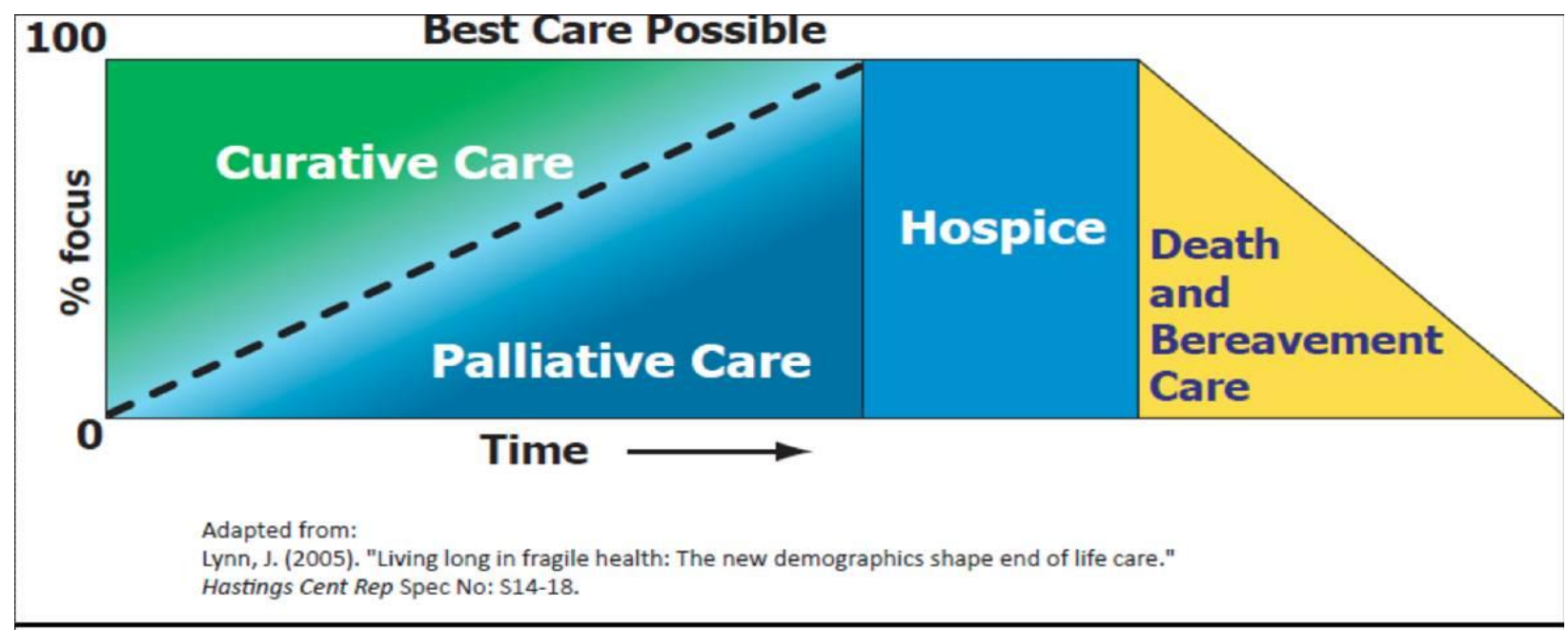

From: Lynn, J. (2005). Living long in fragile health: The new demographics shape end of life 


\section{Appendix B}

Figure 2: Theory of Diffusion of Innovations "S-Shaped Curve"

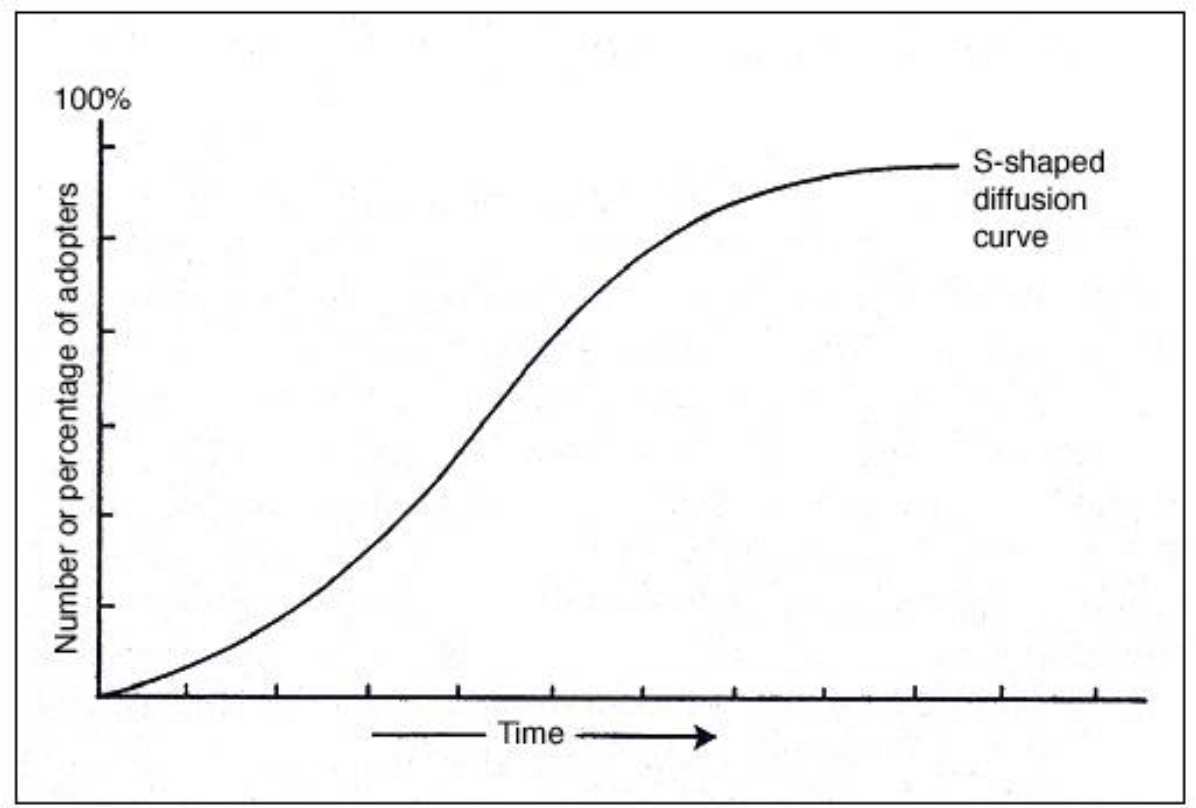

From: Google images: http://johnmill.wordpress.com/archive-2/the-s-shaped-curve/ 


\section{Appendix C}

Table 1: Palliative Care Processes of the Communication and Care Bundle

By ICU Day 1:

(1) Identification of appropriate decision maker

(2) Advance directives

(3) CPR status

(4) Distribution of information leaflet to patient families

(5) Optimal pain assessment

(6) Optimal pain management

By ICU Day 3:

(7) Social work visit

(8) Pastoral care or spiritual support offering

By ICU Day 3:

(9) An interdisciplinary family meeting 


\section{Appendix D}

Figure 3: Supportive Care Patient Information Leaflet
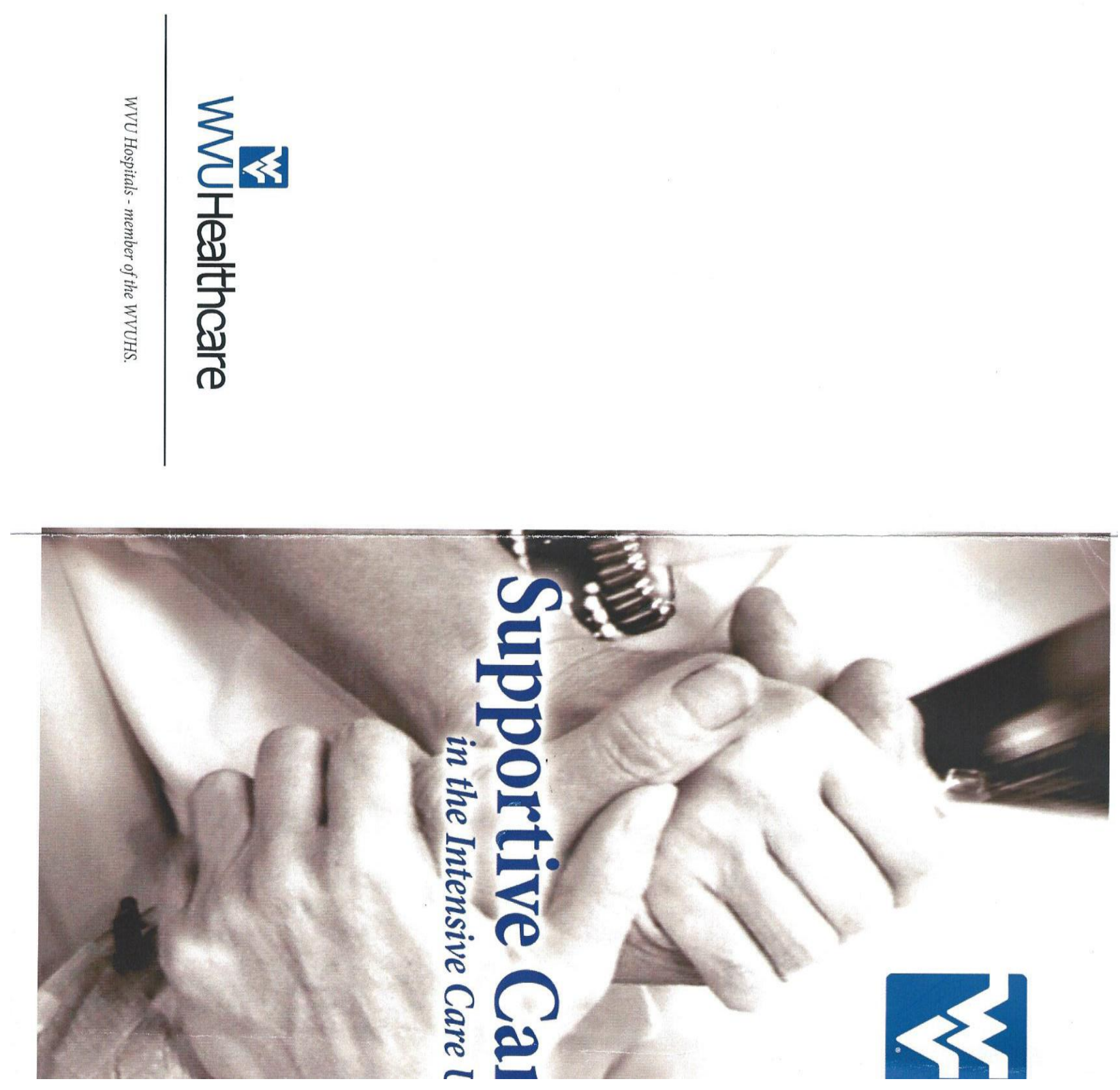

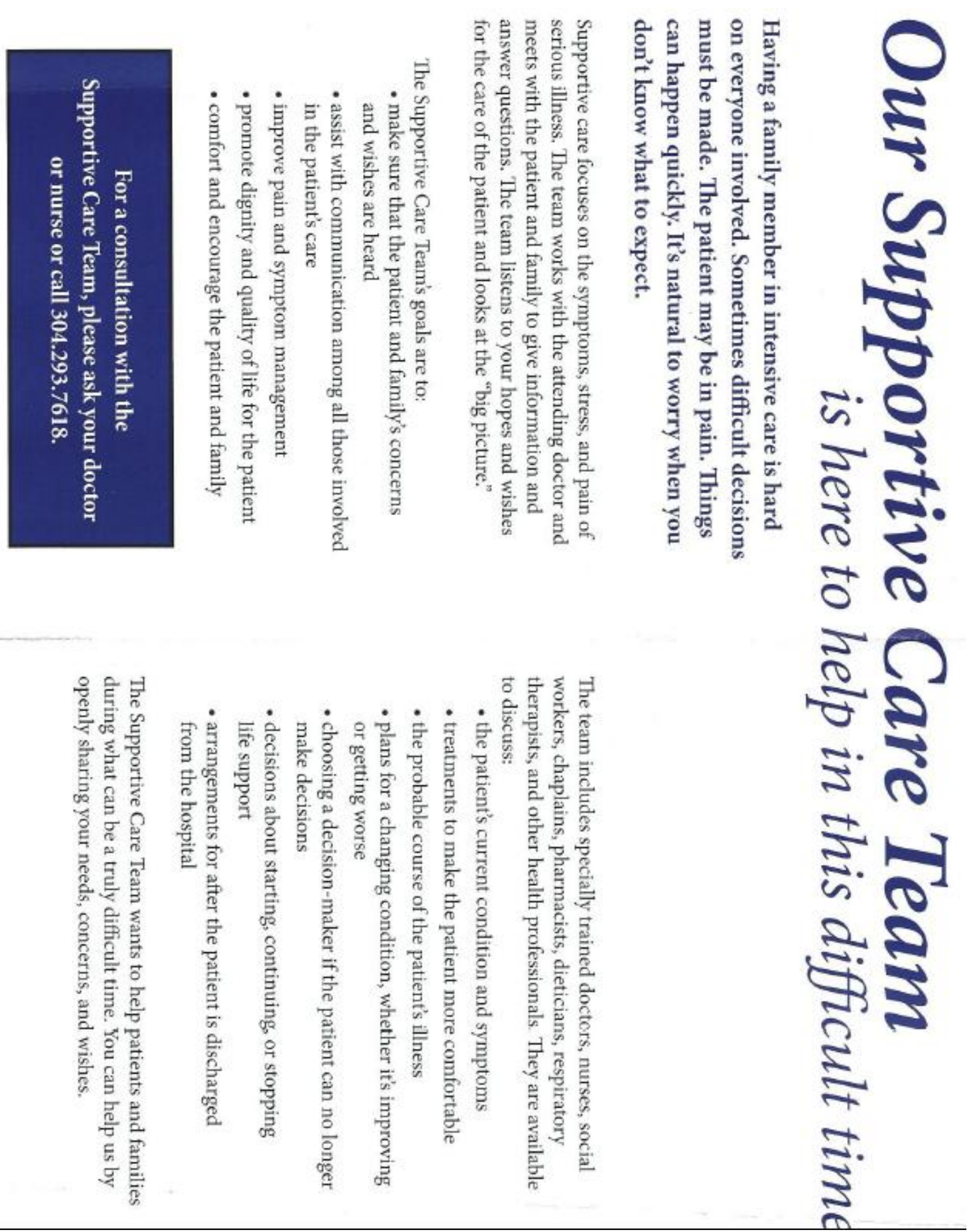


\title{
Appendix E
}

Project Brief

Capstone Proposal Brief: Evaluation of a Nurse Led Intervention to Improve Palliative Care for Medical Intensive Care Patients at High Risk of Death

\author{
Lori A. Constantine, MSN, FNP-BC \\ DNP Student WVU School of Nursing \\ 304-685-4286 or lconstantine@hsc.wvu.edu
}

PROBLEM: One fifth of the patients in the ICU die in or shortly after an ICU stay, most having continual, distressing symptoms.

PICOT: Does the use of the Communication and Care Bundle in a medical/surgical ICU increase the use of palliative processes, and decrease ICU length of stay without increasing mortality for adult medical intensive care patients who are at high risk of death?

\section{Documented palliative processes to be measured are:}

(ICU Admission Day = Day 0. Day 1 starts 24 hours after ICU Admission)

By day 1: Identified appropriate decision maker, advance directives, distribution of information leaflet to patients and/or families, regular pain assessment, and optimal pain management (percentage of 4 hour intervals in which pain rating is $<5$ on a numeric pain scale, $<2$ on The PAIN-AD Scale or $<2$ on the CPOT)

By day 3: Social work visit and spiritual support offered

By day 5: Documentation of an interdisciplinary family meeting

SITE: Combined 18-bed medical ICU and 12-bed surgical ICU in a 521-bed academic medical center in Northern West Virginia

INCLUSION CRITERIA: Adult patients with an ICU length of stay of at least 5 or more days (pre-intervention group only), who were admitted to the MICU service, on either the MICU or SICU physical unit, and identified as being at high risk of death. High risk of death is defined as a patient who meets at least one of the following criteria: (a) ICU admission following a current hospital stay of greater than 10 days (b) Age greater than 80 years in the presence of two or more life-threatening co-morbidities (c) Diagnosis of an active stage IV malignancy (d) Status post cardiac arrest (e) Diagnosis of an intracerebral hemorrhage with a FUNC Score of 4 or less (f) End stage COPD/lung disease/CHF

EXCLUSION CRITERIA: Pregnant persons. Persons less than 18 years old. Persons who do not meet definition for high-risk of death 


\section{PROCEDURES:}

(1) Pre-intervention, obtain baseline data on 9 process measures, mortality, and length of stay

(2) Obtain consent from nurses to participate in educational program

(3) Deliver online educational program, display poster and attend MICU/SICU nursing staff meetings

(4) Six weeks post-intervention, begin post-intervention data collection.

(5) Present findings and publish 


\title{
Appendix F
}

Educational Outline

\section{A Nurse-Led Intervention to Improve Palliative Care for Select Medical ICU Patients}

\section{Outline for Nursing Online Learning Module}

Consent Process - If accepts then will move through module. If declines, user will be prompted to exit the module.

\author{
History of How We Die \\ ○ 100 Years Ago \\ - Pneumonia \\ - Tuberculosis (TB) \\ - Diarrhea or enteritis (CDC, 1999). \\ ○ Now \\ - Cardiovascular disease \\ - Malignant neoplasms \\ - Chronic lower respiratory disease \\ - Cerebrovascular disease \\ - Accidents \\ - Alzheimer's disease \\ - Diabetes \\ (CDC, 2011)
}

\section{Chronic Illness in the US}

- The Problem

- Despite aggressive treatment, many ICU patients die or remain chronically critically ill

○ $20 \%$ of Americans (500,000 people per year) die in or after ICU care

- 100,000 ICU "survivors" continue with critical illness on a chronic basis

- For some critically ill patients, ICU treatment is more burdensome than beneficial and/or inconsistent with their values, goals and preferences

(Angus et al., 2004; Nelson et al., 2010)

\section{The SUPPORT Study}

- 2-year prospective, observational study (phase I) with 4,301 patients followed by a 2-year controlled clinical trial (phase II) with 4,804 patients and their physicians.

- 5 teaching hospitals in the US

- Total sample size of 9,105 adults hospitalized with:

$\circ$ At least 1 of 9 life-threatening diagnoses

○ Overall 6-month mortality rate of 47\% (SUPPORT Investigators, 1996)

\section{SUPPORT STUDY FINDINGS}

- Phase I

○ 53\% MDs did NOT know pt's CPR preference

- $46 \%$ of DNR orders were written within 2 days of death 
- $38 \%$ who died spent at least 10 days in the ICU

○ For $50 \%$ of conscious patients who died in the hospital, family members reported moderate to severe pain at least $50 \%$ of the time (SUPPORT Investigators, 1995)

\section{- Phase II}

○ No increase in patient-physician communication

- No change in 5 targeted outcomes:

- Written DNR orders

- CPR preferences

- LOS in the ICU (receiving mechanical ventilation, or comatose before death)

- Level of reported pain

- Use of hospital resources did not change (SUPPORT Investigators, 1996).

\section{Research Continues to Document Deficiencies in ICU Palliative Care}

- Untreated pain and other symptoms

- Unmet needs for family care

- Inadequate communication

- Conflict among clinicians/patients/families

- Divergence of treatment goals from patient/ family preferences

- Inefficient resource utilization

- Clinician "moral distress" and burnout

\section{7 years of Pain}

- Patient interviews after transfer from surgical intensive care unit:

○ 1990-Puntillo (Heart \& Lung): >70\% recalled pain, 63\% moderate or severe

- 2007-Gélinas (Intensive Critical Care Nurse): 77\% recalled pain, 50\% moderate or severe

\section{Years of Distressing Symptoms}

- Puntillo et al. (2010)-brief surveys of $171 \mathrm{ICU}$

o Most common:

- Tiredness $(75 \%)$

- Thirst (71\%)

- Anxiety (58\%)

○ Most distressing:

- Shortness of breath

- Pain

- Feeling scared or confused

\section{As If that Wasn't Enough....}

- Families DO NOT understand basic information about diagnosis, prognosis, or critical care treatments (Azoulay et al., 2000).

- Family members of ICU patients experience high levels of depression and anxiety (Pochard et al., 2001).

- Survivors of ICU experience high levels of PTSD (Davydow, Gifford, Desai, Needham, \& Bienvenu, 2008). 


\section{Significance in WV}

- $93.27 \%$ multiple chronic illnesses

- Cancer - largest primary diagnosis (37.60\%) followed by neurological diseases (15.20\%)

- $26.68 \%$ patients died while being followed by palliative care services

○ $43.90 \%$ ICU beds

- $58.87 \%$ hospital acute care beds (Emmett, M., Dean, S. \& Kemper, S., 2011)

- $75 \%$ of all West Virginians wish to die at home (WV Center for End-of-Life Care, 2011).

\section{- Significance OUR Academic Medical Center}

- $81.54 \%$ had multiple chronic illnesses

○ $78.46 \%$ for goal clarification

○ $18.46 \%$ for pain and symptom management

○ $3.08 \%$ for psychological or spiritual support (Emmett, Dean, \& Kemper, 2011)

So what is Palliative Care?

- ICU Palliative Care Prioritized for Improvement:

- Institute of Medicine (2001)

- American Thoracic Society (Lankin, et al., 2008)

- American College of Chest Physicians (Selecky, et al., 2005)

- American College of Critical Care Medicine (Truog, et al., 2008)

- National hospital and health care networks, e.g., Voluntary Hospital Association (VHA), Inc. (Center to Advance Palliative Care (CAPC), 2012).

- Veterans Administration Healthcare System (CAPC, 2012)

- Institute for Healthcare Improvement (CAPC, 2012)

- Commercial insurers (CAPC, 2012)

\section{IPAL -ICU Initiative}

\section{Communication and Care Bundle}

- Communication and Care Bundle developed by the VHA, Inc.

- The Communication and Care Bundle began as part of the VHA's "Transformation of the ICU" performance improvement initiative

- Interdisciplinary critical care teams from over 80 hospitals across the country

- Bundle linked to NCP \& NQF's standards and guidelines and its measures are housed in the National Quality Measures Clearinghouse

\section{Communication and Care Bundle By ICU Day 1}

- Identify decision maker

- Address AD status

- Address CPR status

- Distribute info leaflet

- Assess pain regularly

- Manage pain optimally

By ICU Day 3 
- Care Management Contact with Patient and/or Family

- Offer spiritual support

By ICU Day 5

- Interdisciplinary family meeting

\section{The question is....}

- Does the use of the Communication and Care Bundle in a medical ICU increase the use of palliative processes, and decrease ICU length of stay without increasing mortality for select adult MICU service patients?

\section{Inclusion Criteria}

- Adult patients with an ICU length of stay of at least 5 or more days (pre-intervention only), who were admitted to the MICU service and identified as being at high risk of death.

- What defines high risk of death?

- High risk of death is defined as a patient who meets at least one of the following criteria:

- ICU admission following a current hospital stay of greater than 10 days

$\circ$ Age greater than 80 years in the presence of two or more life-threatening comorbidities

- Diagnosis of an active stage IV malignancy

- Status post cardiac arrest

- Diagnosis of an ICH with a FUNC Score of 4 or less

$\circ$ End stage $\mathrm{COPD} /$ lung disease/CHF

\section{Methodology for Review of Literature}

- 5 scholarly databases

- CINAHL, Pub-Med, Education Research Complete, and Health Source Nursing/Academic Edition

- The initial search "Communication and Care Bundle" -3 results

- New Search "palliative care" and "ICU", limited to the past 10 years, peer-reviewed only - 372 hits.

- Search was further narrowed by limiting the years searched to:

○ 2006-2012, peer-reviewed journals only, adult patient population, and ICU setting $=33$ hits.

- Exclusions - not a clinical guideline, systematic review, a quantitative study or a qualitative study $=16$

- Then individually reviewed by the author $=11$ relevant results including those mentioned previously regarding the Communication and Care Bundle.

- Snowballing led to the review of 15 additional articles $=3$ of which were relevant

- Total of 2 clinical guidelines and 13 research studies for review

\section{Results of Review of Literature ... Strategies that improve outcomes...}

- Increase Collaboration 
○ (Billings et al., 2006; Lautrette et al., 2007; Lilly et al., 2000; Nelson, Angus et al., 2006; Nelson, Mulkerin et al., 2006; Nelson, 2010; O’Mahoney et al., 2010; \& Penrod, 2011)

- The use of timely, patient centered, interdisciplinary family meetings

○ (Billings et al., 2006; Lautrette et al., 2007; Lilly et al., 2000; Nelson, Angus, et al., 2006; Nelson, Mulkerin, et al., 2006; Nelson, 2010; \& Penrod, 2011)

- System or staff support including ancillary services such as pastoral care and social services

○ (Billings et al., 2006; Nelson, Angus, et al., 2006; Nelson, Mulkerin et al., 2006; Nelson, 2010; O’Mahoney et al., 2010; \& Penrod, 2011)

- Frequent symptom assessment and management

○ (Billings et al., 2006; Nelson, Angus, et al., 2006; Nelson, Mulkerin, et al., 2006;

O’Mahoney et al., 2010; Penrod, 2011; Pronovost et al., 2012)

- Identification of the patient's medical decision maker

○ (Billings et al., 2006 \& Penrod, 2011)

- Formalization and documentation of advance directives early in the patient's hospitalization

○ (Billings et al., 2010; O’Mahoney et al., 2010; \& Penrod, 2011).

- Brochures for families on bereavement

○ (Billings et al., 2006; Lautrette et al., 2007; Nelson, Angus et al., 2006; \& Nelson, 2010)

- Introduction to palliative care brochure

○ (Nelson, Mulkerin, et al., 2006)

- Education of effective end-of- life communication and other palliative issues

○ (Billings et al., 2006 \& Daly et al., 2010) and

- Open visiting hours

○ (Billings et al., 2006; Nelson, 2010; \& O’Mahoney et al., 2010)

\section{The hypothesis is.....}

- The use of the Communication and Care Bundle in the medical ICU will increase the use of palliative processes, and decrease ICU length of stay without increasing mortality for adult patients who are at high risk of death.

\section{Outputs that are expected....}

- Increased nursing knowledge of their role in palliative care in the ICU

- Increased knowledge of nurse and physician leaders in how palliative processes can be integrated into the medical ICU

- Increase number of palliative care consults

- Outcomes that are expected...

- Increase in the nine palliative care process measures by target time frame

Impacts that are expected...

- Patient care that is more consistent with their values and goals

- Pain is reduced and managed optimally

- Psychosocial and spiritual aspects of care are addressed 
- Family are better informed of their choices for palliative care interventions

\section{BOTTOM LINE}

- I'm a nurse, what does this mean to me?

- There will be a nurse checklist provided to you to stay at the bedside to remind you of your role in implementing the Communication and Care Bundle.

\section{The Checklist}

- Will serve as a reminder of which care processes should occur and by when.

- Take initiative and address each item if they need to be addressed

\section{For example...}

- It is Friday morning before rounds and you notice that Saturday will be the $5^{\text {th }}$ day of your patient's ICU stay.

$\circ$ Remind/request a family meeting by end of the day with the interdisciplinary team

Another example...

- It is Monday. Your patient was admitted on Saturday. The care processes of the Communication and Care Bundle should happen regardless of service or date. If you notice some of the care processes on the checklist have not been attended to, make an effort to get your patient "caught up" and work with what you have

\section{Nurses are the KEY}

- You are the single most important person in your patient's life when it comes to coordinating services, managing care, and addressing physical, psychological, and spiritual needs in the ICU. 


\section{Appendix G}

Figure 4: Poster Displayed in the MICU and SICU

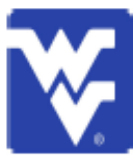

\section{The Problem}

Despite aggressive treatment, many ICU patients die or remain chronically critically ill

$20 \%$ of Americans ( 500,000 people per year) die in or after ICU care

b $100,000 \mathrm{ICU}$ "survivors" contime with critical illness on a chrouic basis

Research Continues to Document Deficiencies in ICU Palliative Care

Untreated pain and other symptoms

- Unmet needs for family care

- Inadequate comminication

- Conflict among clinicians/patients families

- Divergence of treatment goals from patient/ family preferences

- Inefficient resource utilization

> Clinician "moral distress" and burnour

\section{Toward a Solution}

Quality ICU Palliative are has been prioritized for improvement by several national agencies.

Instinte of Medicine (2001)

2. American Thoracic Society (Lankin, et al, 2008)

3. American College of Chest Physicians (Selechy, et al., 2005)

4. American College of Critical Care Medicine (Truog, et al, 2008)

5. National hospital and health care netwodks, e.g. Voluntary Hospital Association (VHA), Inc (Center to Advance Palliative Care (CAPC), 2012).

6. Veterans Administration Healthcare System (CAPC, 2012)

Institute for Healthcare Inprovement (CAPC) 2012)

8. Commercial insurers (CAPC, 2012)
Communication and Care Bundle Palliative Care Integration into the ICU

Lori Constantine, MSN, FNP-BC

West Virginia University School of Nursing, Morgantown, WV 


\section{Appendix $\mathrm{H}$}

\section{Nurses Checklist}

There are nine palliative measures in the Communication and Care Bundle. These nine care processes, which are quality measures, should be documented in the medical record at key time frames.

$\underline{\text { ICU day } 1}$ (the day after ICU admission)

1. Identification of appropriate decision maker

2. Presence of advance directives

3. CPR status that is documented in the electronic medical record (EMR).

4. Documented distribution of information leaflet to patients and/or families in EMR.

5. Ongoing regular pain assessment in EMR. (Regular ongoing pain assessment is at minimum every 4 hours.)

6. Ongoing optimal pain management recorded in EMR. (Optimal pain management is defined by the patient scoring $<5$ on the numeric pain scale, $<2$ on the PAIN$\mathrm{AD}$, or $<2$ on the CPOT.)

\section{$\underline{\text { ICU day } 3}$}

7. Care Management contact documented in the EMR.

8. Spiritual support offered and documented in the EMR

\section{$\underline{\text { ICU day } 5}$}

9. Interdisciplinary family meeting documented in the EMR 


\section{Appendix I \\ Pre-intervention Inclusion Criteria}

\section{Inclusion Criteria}

Adult patients who were admitted to the MICU service and identified as being at high risk of death, who also had an ICU length of stay of five or more days, and physically located on either the MICU or SICU. Patients at high risk of death in the ICU is defined by the literature (Rost et al., 2008; Norton, et. al., 2007) and by local ICU and palliative care experts as meeting one of the criterions listed below. The checked box or boxes is the defining reasons this patient met the inclusion criteria.

1. ICU admission following a current hospital stay of greater than 10 days

2. Age greater than $80 \mathrm{yrs}$ in the presence of two or more life-threatening comorbidities (e.g., end-stage renal disease, severe congestive heart failure)

3. Diagnosis of an active stage IV malignancy

4. Status post cardiac arrest

5. Diagnosis of an intracerebral hemorrhage with a FUNC score of four or less

6. End Stage COPD/lung disease/CHF

\section{Exclusion Criteria}

1. Pregnant persons

2. Persons less than 18 years old

3. Persons who do not meet definition for high-risk of death 


\section{Appendix $\mathbf{J}$}

\section{Post-intervention Inclusion Criteria}

\section{Inclusion Criteria}

Adult patients who were admitted to the MICU service and identified as being at high risk of death, and physically located on either the MICU or SICU. Patients at high risk of death in the ICU is defined by the literature (Rost et al., 2008; Norton, et. al., 2007) and by local ICU and palliative care experts as meeting one of the criterions listed below. The checked box or boxes is the defining reasons this patient met the inclusion criteria.

7. ICU admission following a current hospital stay of greater than 10 days

8. Age greater than $80 \mathrm{yrs}$ in the presence of two or more life-threatening comorbidities (e.g., end-stage renal disease, severe congestive heart failure)

9. Diagnosis of an active stage IV malignancy

10. Status post cardiac arrest

11. Diagnosis of an intracerebral hemorrhage with a FUNC score of four or less 12. End Stage COPD/lung disease/CHF

\section{Exclusion Criteria}

4. Pregnant persons

5. Persons less than 18 years old

6. Persons who do not meet definition for high-risk of death 


\author{
Appendix K \\ Inclusion Criteria Change Approval Letter \\ W. WestVirginiaUniversity. \\ Acknowledgement Letter Amendment \\ From WVU Office of Research Integrity and Compliance \\ Approval Period $\quad 05 / 01 / 2013$ Expiration Date 04/30/2014 \\ Amendment to Approved Protocol \\ Evaluation of Nurse-Led Intervention to Improve Palliative Care for Select \\ Medical Intensive Care Patients
}

To Gina Maiocco

Subject

Protocol Tracking 1302015542A003

Title

Thank you for your submission to the West Virginia University Institutional Review Board IRB.

- IN our initial protocol our inclusion criteria focused on patients who had been admitted to the ICU for at least 5 days. It has come to our attention that patients at high risk for death often die before that 5 th day thus we are not capturing use of palliative care in this patient population. THEREFORE, this amendment requests a change in the inclusion criteria to read: patients admitted to the ICU who meet the definition of high risk of death (the length of stay of 5 days has been removed) The inclusion criteria tool has also been changed to reflect this amendment. A consent waiver is already in place to allow post chart review Use of this inclusion criterion will occur for the post chart data collection period only (nursing education completed and now assessing change in palliative care in the ICU). *The 5 day criterion was only used for the pre chart data collection period.

If you have any questions, please contact the IRB at 3042937073.

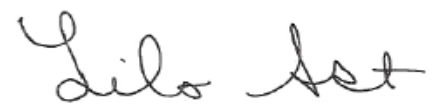

Board Designee Lilo Ast

Letter Sent By Lilo Ast on 08/28/2013 at 15:23:22-04:00 


\section{Appendix L}

\section{Chart Abstraction Tool}

\section{Communication and Care Bundle}

\section{Chart Abstraction Tool}

\begin{tabular}{|c|c|c|c|c|c|c|c|c|c|c|}
\hline \multirow[b]{2}{*}{ Patient Code \# } & \multicolumn{6}{|c|}{ Day 1} & \multicolumn{2}{|c|}{ Day 3} & \multicolumn{2}{|c|}{ Day 5} \\
\hline & $\sum^{\#}$ & $\stackrel{N}{\#}$ & $\underset{0}{\#}$ & & $\sum^{\infty}$ & $\sum^{\infty}$ & 苂 & $\sum_{0}^{\infty}$ & 吾 & 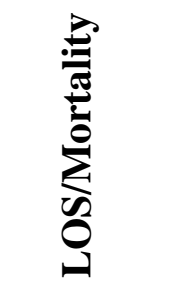 \\
\hline Example & Y & Y & $\mathrm{Y} / \mathrm{b}$ & $\mathrm{N}$ & $6 / 5$ & $6 / 4$ & Y/a & $\mathrm{Y} / \mathrm{a}$ & $\mathrm{Y}$ & $\begin{array}{c}\mathrm{X} \text { days/Y } \\
\text { or } \mathrm{N}\end{array}$ \\
\hline & & & & & & & & & & \\
\hline & & & & & & & & & & \\
\hline & & & & & & & & & & \\
\hline & & & & & & & & & & \\
\hline & & & & & & & & & & \\
\hline & & & & & & & & & & \\
\hline & & & & & & & & & & \\
\hline & & & & & & & & & & \\
\hline & & & & & & & & & & \\
\hline & & & & & & & & & & \\
\hline & & & & & & & & & & \\
\hline & & & & & & & & & & \\
\hline
\end{tabular}




\section{Code Key for Chart Abstraction Tool}

QM \#1: MPOA/Surrogate documented --"Y"/"N"

QM \#2: Advance directives (AD) documented -- "Y"/"N"

QM \#3: Resuscitation Status -- "Y"/"N" If "Y" a) Full Code, b) DNR

QM\#4: Distribution/documentation of ICU info leaflet given -- "Y"/"N"

QM \#5: Regular Pain Assessment- The number of 4 hour intervals eligible for pain assessment over the number of 4 hour intervals with pain assessment documented

QM \#6: Optimal Pain Management- The number of 4hour intervals with documented numerical pain value over the number of 4hour intervals with numeric pain scores $<5,<2$ on the PAIN-AD, or $<2$ on the CPOT.

QM \#7: Documentation that Care Management was contacted either the patient or the family - "Y"/"N" If "Y" offered to a) patient, b) family, c) both

QM\#8: Documentation that Spiritual Support was offered - "Y"/"N" If "Y" offered to a) patient, b) family c) both

QM \#9: Interdisciplinary meeting held on/before Day 5 -- "Y"/"N"/"NA"/"Meeting Refused"; If "Y" meeting with a) patient, b) family c) both

LOS/Mortality - The number of ICU days at either discharge from the ICU or death. Mortality is "Y" for having died or " $\mathrm{N}$ " for living. 


\section{Appendix M}

Table M1:

Project Budget Personnel Costs

\begin{tabular}{|c|c|c|c|}
\hline Role & Department & Hours Spent & Base salary \\
\hline Co-PI (myself) & Student & $16 \times 20$ weeks & $\$ 50 /$ hour $=\$ 16,000$ \\
\hline Capstone Chair (PI) & SON & 1 x 20 weeks & $\$ 75 /$ hour $=\$ 1,500$ \\
\hline Capstone Member & Project Site & 12 & $\$ 75 /$ hour $=\$ 900$ \\
\hline Project Manager & Project Site & 6 & $\$ 75 /$ hour $=\$ 450$ \\
\hline Statistician & SON & 6 & $\$ 75 /$ hour $=\$ 450$ \\
\hline Net Learning Specialist & Project Site & 4 & $\$ 50 /$ hour $=\$ 400$ \\
\hline Palliative Care MD Expert & Project Site & $1 \mathrm{MD}-8$ & $\$ 150 /$ hour $=\$ 1,200$ \\
\hline & & $1 \mathrm{MD}-2$ & $\$ 150 /$ hour $=\$ 300$ \\
\hline Leadership Experts & Project Site & & \\
\hline ICU Director & & 4 & $\$ 75 /$ hour $=\$ 300$ \\
\hline ICU Managers & & (2) at 8 each & $\$ 65 /$ hour $=\$ 1,040$ \\
\hline Care Management Director & & (1) at 4 & $\$ 75 /$ hour $=\$ 300$ \\
\hline Pastoral Care Director & & (1) at 2 & $\$ 75 /$ hour $=\$ 150$ \\
\hline Total & & & $\$ 22,990$ \\
\hline
\end{tabular}

*Total human costs were integrated into current roles of the persons listed above.

Table M2:

Project Budget Equipment/Supplies Costs

\begin{tabular}{lccc}
\hline \multicolumn{1}{l}{ Item } & Quantity & Cost Each & $\begin{array}{c}\text { Total Cost with } \\
0.06 \% \text { sales tax }\end{array}$ \\
SPSS software & 1 & $\$ 90.00$ & $\$ 95.40$ \\
Lamination & 32 & $\$ 1.00$ & $\$ 33.92$ \\
Paper & 1 ream & $\$ 6.00$ & $\$ 6.36$ \\
Ink & 1 cartridge & $\$ 30.00$ & $\$ 31.80$ \\
Professional Poster & 3 & $\$ 11.00$ & $\$ 34.98$ \\
Total & & & $\$ 202.46$ \\
\hline
\end{tabular}




\section{Appendix N}

\section{Hospital Approval Letter}

WestVirginiaUniversityHospitals

February 12, 2013

Lori Constantine, MSN, FNP-BC

DNP Student

WVU School of Nursing

Morgantown, WV 26506

Dear Lori,

This is to acknowledge receipt of your request in regards to the conduct of research entitled "Evaluation of a Nurse Led Intervention to Improve Palliative Care for Medical Intensive Care Patients at High Risk of Death" to be conducted at West Virginia University Hospitals in the medical and surgical ICU. My response is in the affirmative; you are granted permission to conduct your research, with the following stipulations:

1) Permission is granted based on the research being carried out precisely as defined in your methodology;

2) Permission is granted contingent upon approval and/or recommendations of the WVU Institutional Review Board;

3) At the completion of the study, you are requested to share your findings with those most concerned with the outcomes.

Best wishes to you in this endeavor!

Cordially,

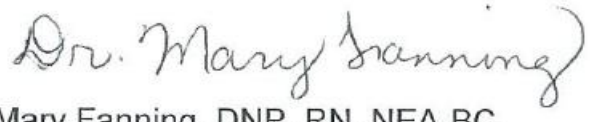

Mary Fanning, DNP, RN, NEA-BC

Director, Nursing Administration / Magnet Project Director

WVU Healthcare

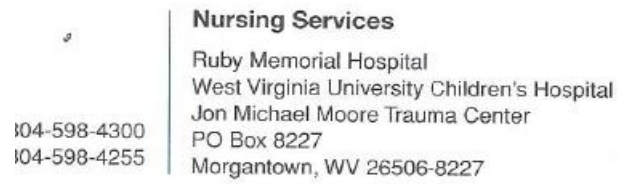

Equal Opportunity/Affirmative Action Institution 


\section{Appendix $\mathrm{O}$}

IRB Approval Letter

\section{WWestVirginiaUniversity,}

\section{Approval Letter - Expedited}

To: $\quad$ Gina Maiocco

From:

WVU Office of Research Integrity and Compliance

Approval Period:

$05 / 01 / 2013$

Expiration Date: $\quad 04 / 30 / 2014$

Subject:

Protocol Approval Letter

Protocol Tracking\#: 1302015542

Title:

Evaluation of Nurse-Led Intervention to Improve Palliative Care for Select Medical Intensive Care Patients

The research study referenced above was reviewed by the West Virginia University Institutional Review Board (IRB) via expedited review procedures and was approved in accordance with 46 CFR 46.101(b).

The documents listed below have been approved and validated for use in this study and are available in the WVU+kc system.

- $\quad$ categories 5 and 7

Thank you.

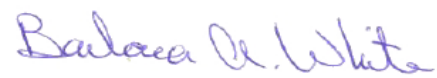

Board Designee: Barbara White

Letter Sent By: Barbara White on 05/01/2013 at 11:46:05-04:00

Once you begin your human subject research, the following regulations apply:

1. Unanticipated or serious adverse events/side effects encountered in this research study must be reported to the IRB within five (5) days. 
2. Any modifications to the study protocol or informed consent form must be reviewed and approved by the IRB prior to implementation.

3. You may not use a modified informed consent form until it has been approved and validated by the IRB. 


\section{Appendix P}

Table P1:

Quality Measure Frequencies both Pre-Intervention and Post-Intervention as Documented by Day 1

Quality Measure

\section{Advance directives}

No

Yes

MPOA or Surrogate

No

Yes

\section{Resuscitation Status}

No

Yes, Full Code

Yes, DNR

Patient Leaflet

$$
\text { No }
$$

Yes
Pre-Intervention

Frequency/Percent Frequency/Percent

$33(60 \%)$

$22(40 \%)$

$33(60 \%)$

$22(40 \%)$

$0(0 \%)$

$54(98.2 \%)$

$1(1.8 \%)$

$0(0 \%)$

$0(0 \%)$
$16(29.1 \%)$

$39(70 \%)$

$\mathrm{Chi}^{2}$

Value

10.636

0.001

10.636

$16(29.1 \%)$

$39(70.9 \%)$

$0(0 \%)$

$47(85.5 \%)$

$8(14.5 \%)$

5.930

0.015
Sig.

(n) 
Table P3:

Quality Measure Frequencies both Pre-Intervention and Post-Intervention as Documented by Day 3

Quality Measure

Care Management Visit

No

Yes with patient only

Yes with family only

Yes with both patient and

family

Total Care Management

Visits
Pre-Intervention Post-Intervention $\quad \mathrm{Chi}^{2}$ Value Sig.

Frequency/Percent Frequency/Percent

$\begin{array}{ll}31(56.4 \%) & 24(43.6 \%) \\ 8(14.5 \%) & 9(16.4 \%) \\ 6(10.9 \%) & 18(32.7 \%) \\ 10(18.2 \%) & 4(7.3 \%) \\ 24(43.6 \%) & 31(56.4 \%)\end{array}$

9.521

0.023

Spiritual Support Offered

No

Yes with patient only

Yes with family only

Yes with both patient and

family

Total times spiritual support $12(21.8 \%)$

$43(78.2 \%)$

$36(65.5 \%)$

$3(5.5 \%)$

$8(14.5 \%)$

$5(9.1 \%)$

$1(1.8 \%)$

$6(10.9 \%)$

$8(14.5 \%)$

$19(34.5 \%)$ offered

\footnotetext{
*Items in bold were found to be significant.
}

Table P4:

Quality Measure Frequencies both Pre-Intervention and Post-Intervention as Documented by Day 5

\begin{tabular}{clllc}
\hline \multicolumn{1}{c}{ Quality Measure } & $\begin{array}{l}\text { Pre-Intervention } \\
\text { Frequency/Percent }\end{array}$ & $\begin{array}{l}\text { Post-Intervention } \\
\text { Frequency/Percent }\end{array}$ & Chi $^{2}$ Value & Sig. \\
Interdisciplinary Family Meeting & & & & \\
No & $35(63.6 \%)$ & $37(67.3 \%)$ & \multirow{2}{*}{2.523} & 0.471 \\
Yes with patient only & $3(5.5 \%)$ & $6(10.9 \%)$ & & \\
Yes with family only & $12(21.8 \%)$ & $10(18.2 \%)$ & & \\
Yes with both patient and & $5(9.1 \%)$ & $2(3.6 \%)$ & \\
family & $20(36.4 \%)$ & $18(32.7 \%)$ & \\
Total family meetings & & & \\
\hline
\end{tabular}


Table P5:

Patient Outcome ICU Length of Stay both Pre-Intervention and Post-Intervention

\begin{tabular}{cccccc}
\hline Outcome & $\mathrm{N}$ & Mean & $\begin{array}{c}\text { Standard } \\
\text { Deviation }\end{array}$ & $\mathrm{T}$ & $\begin{array}{c}\text { Sig. } \\
\text { (2-tailed) }\end{array}$ \\
ICU Length of Stay & & & & & \\
Pre-Intervention & 55 & 10.69 & 8.322 & 3.859 & $<\mathbf{0 . 0 1}$ \\
Post-Intervention & 55 & 4.89 & 7.415 & \\
\hline
\end{tabular}

*Items in bold were found to be significant.

Table P6:

Patient Outcome Mortality both Pre-Intervention and Post-Intervention

Outcome Pre-Intervention Post--Intervention $\mathrm{Chi}^{2}$ Value Sig.

Mortality

No

Died in the ICU

Died After ICU

Discharge

$\begin{array}{lccc}29(52.7 \%) & 38(69.1 \%) & & \\ 12(21.8 \%) & 10(18.2 \%) & 3.724 & 0.155 \\ 14(25.5 \%) & 7(12.7 \%) & & \end{array}$


Table P7:

Patient Population with the Specified Inclusion Criteria both Pre-Intervention and PostIntervention

Inclusion Criteria Frequency/Percent

$\mathrm{Chi}^{2}$ Value

Sig.

ICU Admission following a current hospital stay of more than 10 days

Pre-Intervention

Post-Intervention

Age $>80$ years in the presence of 2 or more

life-threatening co-morbidities

Pre-Intervention

Post-Intervention

Diagnosis of an Active Stage IV Malignancy

Pre-Intervention

Post-Intervention

Status Post Cardiac Arrest

Pre-Intervention

Post-Intervention

Diagnosis of an ICH with a FUNC score of 4

or less

Pre-Intervention

Post-Intervention

End Stage COPD/Lung Disease/CHF

Pre-Intervention

Post-Intervention
0.340

0.909

$4(7.3 \%)$

$8(14.5 \%)$

3.411

0.065

$16(29.1 \%)$

$3(5.5 \%)$

1.089

0.297

$6(10.9 \%)$

$11(20.0 \%)$

0.573

0.449

$8(14.5 \%)$

$0(0.0 \%)$

3.084

0.079

$3(5.5 \%)$

$36(65.5 \%)$

5.280

0.022

*Items in bold were found to be significant. 


\section{Table P8:}

Nurse Demographics Obtained During On-Line Educational Module

Nurse Characteristics

Nurses Age in Years

$$
\begin{aligned}
& 20-30 \\
& 31-40 \\
& 41-50 \\
& >50
\end{aligned}
$$

Highest Nursing Degree Obtained

$$
\begin{aligned}
& \text { AND } \\
& \text { Diploma } \\
& \text { BSN } \\
& \text { MSN }
\end{aligned}
$$

Years Employed at Hospital

$$
<5 \text { years }
$$$$
\text { 6-10 years }
$$$$
\text { 11-20 years }
$$$$
>20 \text { years }
$$

Years Nursing Experience

$$
<5 \text { years }
$$

6-10 years

11-20 years

$>20$ years

Years ICU Experience

$<5$ years

6-10 years

11-20 years

$>20$ years

Specialty Certification

CCRN

Other Critical Care Certification

Other General Certification

None

Gained New Knowledge

Yes, Definitely

Yes, Mostly

No, Not Really

Absolutely Not
Frequency

Percent

$56.7 \%$

$21.1 \%$

$12.2 \%$

$10.0 \%$

9

17

$18.9 \%$

1

70

2

58

11

15

6

55

11

13

11

57

13

11

9

$1.1 \%$

$77.8 \%$

$2.2 \%$

$64.4 \%$

$12.2 \%$

$16.7 \%$

$6.7 \%$

$61.1 \%$

$12.2 \%$

$14.4 \%$

$12.2 \%$

$63.3 \%$

$14.4 \%$

$12.2 \%$

$10.0 \%$

15

$16.7 \%$

6

3

66

$6.7 \%$

$3.3 \%$

$73.3 \%$

30

49

$33.3 \%$

$54.4 \%$

10

$11.1 \%$ 


\section{Table P9:}

Nurse Perspectives Related to Palliative Care in the ICU

Nurse Perspective Frequency Percent

Biggest Obstacle for Palliative Care in the ICUs

Lack of Communication Among the Healthcare Team 34

Lack of Communication with Families

$37.8 \%$

Knowledge

Time

$13 \quad 14.4 \%$

Other

Biggest Area for Improvement in Palliative Care in the ICUs

Lack of Communication Among Patients, Families, and the Healthcare Team

$7 \quad 7.8 \%$

$11 \quad 12.2 \%$ 


\section{Appendix Q}

Recommendations

1. The MICU and SICU at this academic medical center should consider the adoption of a policy that advance directives are scanned on the first day of ICU admission, or as soon as they become available.

2. An interdisciplinary team at this academic medical center should examine the potential for a new, electronic method for capturing end-stage diagnoses, so that it is readily visible in the patient's EMR.

3. Future research should be initiated to determine if there is a difference code status in patients with diagnoses of end-stage COPD/lung disease/CHF, that may be correlated with surviving past ICU admissions; thereby impacting end-of-life preferences.

4. Further discussion in the ICU should reflect upon making life-limiting illness more recognizable at a glance, so patient care and goals can be better aligned. For example: Discuss how the surprise question can be used in the ICU to trigger a palliative care consult, or an interdisciplinary team meeting focused on goal clarification.

5. Future conversations among the Supportive Care and ICU interdisciplinary team is warranted to ways to facilitate interdisciplinary meetings for patients at high risk of death on or before day five of their ICU stay.

6. Careful review of potential contributing factors as why there was no documentation of the Supportive Care Patient Information Leaflet should be thoroughly explored. Consultation with Information Technology may facilitate the documentation of this quality measure within the nurses' documentation flow sheets in the EMR.

7. Future research using the same pre-intervention data should be compared with new postintervention data, incorporating inclusion criteria of at least five days, as in the preintervention period.

8. Inquiry is needed to further define the nurses' perspectives related to obstacles for palliative care in the ICU.

9. Inquiry as to if further education may be needed for the ICU staff in treating pain measured via observational pain scales is worth consideration. 


\section{References}

Achterberg, T., Schoonhoven, L., \& Grol, R. (2008). Nursing Implementation Science: How evidence-based nursing requires evidence-based implementation. Journal of Nursing Scholarship, 302-310.

American Nurses Credentialing Center. (2012). Forces of magnetism. Retrieved from the American NursesCredentialing Center website:

http://www.nursecredentialing.org/Magnet/ProgramOverview/HistoryoftheMagnetProgra $\underline{\mathrm{m} / \text { ForcesofMagnetism.aspx }}$

Angus, D.C., Barnato, A.E., Linde-Zwirble, W., Weissfeld, L., Watson, R., Rickert, T., \& Rubenfeld, G. (2004). Use of intensive care at the end of life in the United States: an epidemiologic study. Critical Care Medicine, 32(3), 638-643.

Appalachian Regional Commission. (2011). Data reports. Retrieved from Appalachian Regional Commission website: http://www.arc.gov/index.asp.

Arbour, C. \& Gelinas, C. (2011). Setting goals for pain management when using a behavioral scale: Example with the Critical-Care Pain Observation tool. Critical Care Nurse, 31, 66-68 doi: $10.4037 / \operatorname{ccn} 2011914$

Arbour, C., Gelinas, C., Mauchad, C. (2011). Impact of the implementation of the Critical-Care Pain Observation Tool (CPOT) on pain management clinical outcomes in mechanically ventilated trauma intensive care unit patients: A pilot study. Journal of Trauma Nursing, $181(1), 52-60$.

Azoulay, E., Chevret, S., Leleu, G. Pochard, F., Barboteu, M., Adrie, C., ...\& Schlemmer, B. (2000). Half the families of ICU patients experience inadequate communication with physicians. Critical Care Medicine,8, 3044-3049. 
Barnett, M.M. (2002). Effect of breaking bad news on patients' perceptions of doctors. Journal of the Royal Society of Medicine, 95(7), 343-347.

Brouwers, M., Kho, M.E., Browman, G.P., Burgers, J.S., Cluzeau, F., Feder, G., ...\& Zitzelsberger, L. for the AGREE Next Steps Consortium. (2010). AGREE II: Advancing guideline development, reporting and evaluation in healthcare. Canadian Medical Association Journal. Available online July 5, 2010. doi:10.1503/cmaj.090449

Behringer, B. \& Friedell, G. (2006). Appalachia: where place matters in health. Prevention of Chronic Disease, 3(4), A113. Retrieved from http://www.ncbi.nlm.nih.gov/pmc/articles/PMC1779277/

Billings, J.A., Keeley, A., Bauman, J., Cist, A., Coakley, E., Dahlin, C.,...Massachusetts General Hospital Palliative Care Nurse Champions. (2006). Critical Care Medicine 34(11), S388S393.

Bradley, E.H., Prigerson, H., Cherlin, E., Johnson-Hurzeler, R., \& Kasi, S.V. (2002). Delayed hospice enrollment and caregiver well-being. Gerontologist, 254, 254.

Breen, C.M., Abernethy, A.P., Abbott, K.H. (2001). Conflict associated with decisions to limit life-sustaining treatment in intensive care units. Journal of General Internal Medicine, 16, 283-289.

Boyd, D., Merkh, K., Rutledge, D.N., \& Randal, V. (2011). Nurses' perceptions and experiences with end-of-life communication and care. Oncology Nurse Forum, 38(3), E229-39.

CAMC Health Education and Research Institute. (2010). West Virginia palliative care team report. Retrieved from West Virginia Center for End of Life Care website:. http://www.wvendoflife.org/MediaLibraries/WVCEOLC/Media/palliative\%20care\%20ne twork\%20reports/2010PalliativeCareNetworkReport.pdf 
CAMC Health Education and Research Institute. (2009). West Virginia palliative care team report. Retrieved from West Virginia Center for End of Life Care website: http://www.wvendoflife.org/MediaLibraries/WVCEOLC/Media/palliative\%20care\%20ne twork\%20reports/2009PalliativeCareNetworkReport.pdf

Candrilli, S. \& Mauskopf, J. (2006, May 20-24). How much does a hospital day cost?

Poster session presented at 11th Annual International Meeting of the International Society for Pharmacoeconomics and Outcomes Research, Philadelphia, PA.

Centers for Disease Control. (2005). Deaths: final data for 2005. Vitals Statistics Reports 56(10), p. 1 .

Centers for Disease Control. (1999). Achievements in public health, 1900-1999: Control of infectious diseases. $M M W R, 48(29), 621-629$.

Centers for Disease Control. (2008). West Virginia: The burden of chronic disease. Retrieved from the Center for Disease Control website: http://www.cdc.gov/chronicdisease/states/pdf/west_virginia.pdf

Centers for Disease Control. (2011). Deaths: preliminary data from 2011. National Vitals Statistics Reports, 61(6), p. 6.

Center to Advance Palliative Care. (2012). The IPAL project: Improving palliative care. Retrieved from the Center to Advance Palliative Care website: http://www.capc.org/ipal/ipal-icu/professional-organizations

Childers, J.W., Arnold, R., \& Curtis, J.R. (2005). Prognosis in end-stage COPD: Fast facts and concepts \#141. Available at: http://www.eperc.mcw.edu/EPERC/FastFactsIndex/ff_141.htm. 
Chiplasky, L.M. (2013). End-of-life care: Are nurses educationally prepared? RN Journal of Nursing. Available at: http://rnjournal.com/journal-of-nursing/end-of-life-care-arenurses-educationally-prepared

Clarke, E.B., Curtis, J.R, Luce, J.M., Levy, M., Danis, M., Nelson, J., ...\& Robert Wood Johnson Foundation Critical Care End-Of-Life Peer Workgroup Members. (2003). Quality indicators for end-of-life care in the intensive care unit. Critical Care Medicine, 31(9), 2255 -2262.

Cloud, J. (2000). A kinder, gentler death. Time Magazine. Retrieved from: http://www.time.com/time/magazine/article/0,9171,997968,00.html

Cohen, L. M., Ruthazer, R., Moss, A.H., Germain, M.J. (2010). Predicting six-month mortality for patients who are on maintenance hemodialysis. Clinical Journal of the American Society of Nephrology, 5, 72-79.

Cook, D.J., Guyatt, G., Rocker, G., Sjokvist, P., Weaver, B., Dodek, P, ..\& Cook, R. (2001). Cardiopulmonary resuscitation directives on admission to intensive-care unit: an international observational study. Lancet, 358, 1941-1945.

Communication and Care Quality Measures at the National Quality Measures Clearinghouse sponsored by the Agency for Healthcare Research and Quality. Retrieved from the National Quality Measures Clearinghouse website: http://www.qualitymeasures.ahrq.gov. Accessed November 4, 2012.

Curtis, J.R., \& Rubenfeld, G.D. (2005). Improving palliative care for patients in the intensive care unit. Journal of Palliative Medicine, 8, 840-854.

Curtis, J., Treece, P., Nielsen, E., Downey, L., Shannon, S., Braungardt, T.,... \& Engelberg, R. (2008). Integrating palliative and critical care: evaluation of a quality improvement 
intervention. American Journal of Respiratory \& Critical Care Medicine, 178(3), 269275.

Curtis, J., Nielsen, E., Treece, P., Downey, L., Dotolo, D., Shannon, S., ...\& Engelberg, R. (2011). Effect of a quality-improvement intervention on end-of-life care in the intensive care unit: A randomized trial. American Journal of Respiratory \& Critical Care Medicine, 183(3), 348-355. doi:10.1164/rccm.201006-1004OC

Dartmouth Atlas of Healthcare. (2011). Trends and variation in end-of-life care for Medicare beneficiaries with severe chronic illness. Retrieved from the Dartmouth Atlas of Healthcare website: http://www.dartmouthatlas.org/downloads/reports/EOL_Trend_Report_0411.pdf

Davydow, D.S., Gifford, J.M., Desai, S.J., Needham, D.M., \& Bienvenu, O.J. (2008). Posttraumatic stress disorder in general intensive care unit survivors: a systematic review General Hospital Psychiatry 30(5), 421-434. doi: 10.1016/j.genhosppsych.2008.05.006

Demaio, A. (2012). Global Health in the 21st Century: Non-Communicable Diseases - A presentation prepared by Dr Alessandro, for the 2012 Global Health Conference, Cairns, Australia. Copenhagen School of Global Health, Denmark. Retrieved from the World Health Organization website: http://prezi.com/-f8htiqax3rq/global-health-in-the-21stcentury-non-communicable-diseases-abbrev/

Desbiens, N.A., Wu, A.W., \& Broste, S.K. (1996). Pain and satisfaction with pain control in seriously ill hospitalized adults: findings from the SUPPORT research investigations. Critical Care Medicine, 24, 1953-1961.

Emmett, M., Dean, S. \& Kemper, S. (2013). WV palliative care team report. Retrieved from the West Virginia Center for End of Life Care website: 
http://www.wvendoflife.org/MediaLibraries/WVCEOLC/Media/palliative\%20care\%20ne twork\%20reports/2012PalliativeCareNetworkReport.pdf

Guyatt, G., Rocker, G., Marshall, J., Leasa, D., Levy, M., Varon,, J., ...\& Hamel, M.B. (2000). Decision-making and outcomes of prolonged ICU stays in seriously ill patients. Journal of American Geriatric Society, 48, 70-74.

Institute for Clinical Systems Improvement (2011). Palliative care for adults (4th Ed.). Available at: https://www.icsi.org/_asset/k056ab/PalliativeCare.pdf

Institute of Medicine. (2001). Crossing the quality chasm: a new health system for the $21^{\text {st }}$ century. Washington D.C., National Academy Press.

The Joint Commission. (2008). Comprehensive Accreditation Manuals for Hospitals: The Official Handbook. Oakbrooke Terrace, Il: The Joint Commission.

Keenan, S.P., Busche, K.D., Chen, L.M. (1997). A retrospective review of a large cohort of patients undergoing the process of withdrawing or withholding of life support. Critical Care Medicine, 25, 1324-1331.

Lanken, P.N., Terry, P.B., Delisser, H.M., Fahy, B.F., Hansen-Flaschen, J., Heffner, J.E., ...\& ATS End-of-Life Care Task Force. (2008). An official American Thoracic Society clinical policy statement: palliative care for patients with respiratory diseases and critical illnesses. American Journal Respiratory Critical Care Medicine, 177, 912-927.

Larrabee, J. H. (2009). Nurse to Nurse: Evidence-Based Practice. New York, NY: McGraw-Hill, p. 125 .

Lautrette, A., Darmon, M., Megarbane, B., Joly, L.M., Chevret, S., Adrie, C., ...\& Azoulay E. (2007). A communication strategy and brochure for relatives of patients dying in the ICU. New England Journal of Medicine 365(5), 469-478. 
Lilly, C.M., De Meo, D.L., Sonna, L.A., Haley, K.J., Massaro, A.F., Wallace, R.F., \& Cody, S. (2000). An intensive communication intervention for critically ill. American Journal of Medicine, (109), 469-475.

Luce, J.M. \& Prendergast, T. J. (2001). The changing nature of death in the ICU. In: J. R. Curtis \& G. D. Rubenfeld (Eds). Managing death in the intensive care unit: the transition from cure to comfort (pp. 19-29). Oxford, UK, Oxford University Press.

Lynn, J. (2005). "Living long in fragile health: The new demographics shape end of life care." Hastings Center Special Report No: S14-18.

Meier, D.E., Tuch, H., Campbell, M., Keyserling, J., \& Morrison, R.S. (2009). Improving quality, reducing cost through increased access to palliative care: Recommendations for health care reform. Submitted June 10, 2009 on behalf of the Hospice and Palliative Care Coalition.

Menon, A., Korner-Bitensky, N., Kastner, M., McKibbon, K. \& Straus, S. (2009). Strategies for rehabilitation professionals to move evidence-based knowledge into practice: a systematic review. Journal of Rehabilitation Medicine (Stiftelsen Rehabiliterings Information), 41(13), 1024-1032.

Milken Institute. (2011). An unhealthy America: The economic burden of chronic disease. report. Retrieved from the Milken Institute website: http://www.milkeninstitute.org/pdf/chronic_disease_report.pdf

Morrison, R.S. (2009). Palliative care: Policy initiatives to improve access, improve quality, and reduce costs. Retrieved from the National Palliative Care Research Center website: http://www.npcrc.org/ 
Morrison, R.S., Dietrich, J., Ladwig, S., Quill, T., Sacco, J., Tangeman, J. \& Meier, D. (2011). Palliative care consultation teams cut hospital costs for Medicaid beneficiaries. Health Affairs (30), 3, 454-463. doi: 10.1377/hlthaff.2010.0929

Moss, A.H., Demanelis, A.R., Murray, J., \& Jack, J. (2005). Barriers to quality end-of-life care in West Virginia ICU units: physicians' and nurses' perspectives. WV Medical Journal. $101,(5), 200-204$.

Moss, A. H., Ganjoo, .J, Sharma, S., Gansor, J., Senft, S., Weaner, B., ...\& Schmidt, R. (2008). Utility of the "surprise" question to identify dialysis patients with high mortality. Clinical Journal of the American Society of Nephrology, 3, 1379-1384.

Moss, A. H., Lunney, J. R., Culp, S., Auber, M., Kurian, S., Rogers, J., ..\&\& Abraham, J. (2010). Prognostic significance of the "surprise" question in cancer patients. Journal of Palliative Medicine, 13, 837 - 840.

Mularski, R.A., Curtis, J.R., Billings, J.A., Burt, R., Byock, I., Fuhrman, C., ...\& Levy, M.M. (2006). Proposed quality measures for palliative care in the critically ill: A consensus from the Robert Wood Johnson Foundation Critical Care Workgroup. Critical Care Medicine 34(11), S404-S411.

National Cancer Institute. (2013). Cancer staging fact sheet. Retrieved from: National Cancer Institute website: http://www.cancer.gov/cancertopics/factsheet/detection/staging National Consensus Project for Quality Palliative Care. (2013). Clinical guidelines for quality palliative care. Retrieved from: National Consensus Project for Quality Palliative Care website: http://www.nationalconsensusproject.org

National Hospice and Palliative Care Organization. (2010). Position Statement and call to action on access to palliative care in critical care settings. Retrieved from the National Hospice 
and Palliative Care website: http://www.nhpco.org/files/public/NHPCO_PC-in-

ICU_statement_Sept08.pdf

National Quality Forum. (2011). Framework and preferred practices for quality palliative and hospice care. Retrieved from the National Quality Forum website: www.qualityforum.org/publications/reports/palliative.asp

Nelson, J.E., Angus, D.C., Weissfeld, L.A., Puntillo, K.A., Danis, M., Deal, D...\&\& Cookj, D.J. (2006). End-of-life care for the critically ill: A national intensive care unit survey. Critical Care Medicine, 34(10), 2547-2553.

Nelson, J., Bassett, R., Boss, R., Brasel, K., Campbell, M., Cortez, T., ...\& Weissman, D. (2010). Models for structuring a clinical initiative to enhance palliative care in the intensive care unit: a report from the IPAL-ICU Project. Critical Care Medicine, 38(9), 1765-1772. doi:10.1097/CCM.0b013e3181e8ad23

Nelson, J.E., Meier, D.E. \& Oei, E.J. (2001). Self-reported symptom experience of critically ill cancer patients receiving intensive care. Critical Care Medicine, 29, 277-282.

Nelson, J.E., Mulkerin, C.M., Adams, L.L., \& Pronovost, P.J. (2006). Improving comfort and communication in the ICU: A practical new tool for palliative care performance measurement and feedback. Quality Safety in Health Care 15, 264-271.

Nelson, J.E., Puntillo, K.A., Pronovost, P.J., Walker, A.S., McAdam, J.L., Ilaoa, J., ...\& Penrod, J. (2010). In their own words: Patients and families define high-quality palliative care in the intensive care unit. Critical Care Medicine 38(3), 808-818.

Ngo-Metzger, Q., Augus, K.J., Srinivasan, M., Liao, S., Meyskens, F.L. (2008). End-of-Life care: guidelines for patient-centered communication. American Family Physician, 77(2), $167-174$. 
Norton, S.A., Hogan, L.A., Holloway, R.G., Temkin-Greener, H. Buckley, M.J., \& Quill, T.E. (2007). Proactive palliative care in the medical intensive care unit: effects on length of stay for selected high-risk patients. Critical Care Medicine (35), 6, 1530-1535.

O'Brien, M.A., Rogers, S., Jamtvedt, G. (2007). Educational outreach visits: effects on professional practice and health care outcomes. Cochrane Database Systematic Reviews (4), CD000409.

O'Mahony, S., McHenry, J., Blank, A., Snow, D., Karakas, S., Santoro, G., ... \& Kvetan, V. (2010). Preliminary report of the integration of a palliative care team into an intensive care unit. Palliative Medicine, 24(2), 154-165. doi:10.1177/0269216309346540

Pattison, M. \& Romer, A. L. (2001). Improving care through the end of life: launching a primary care clinic-based program. Journal of Palliative Medicine, 4, 249-254.

Penrod, J.D., Luhrs, C.A., Livote, E.E., Cortez, T.B., \& Kwak, J. (2011). Implementation and evaluation of a network-based pilot program to improve palliative care in the intensive care unit. Journal of Pain and Symptom Management, 42(5), 668-671.

Penrod, J.D., Pronovost, P.J., Livote, E.E., Puntillo, K.A., Walker, A.S., Wallenstein, S., ...\& Nelson, J.E. (2012). Meeting standards of high-quality intensive care unit palliative care: Clinical performance and predictors. Critical Care Medicine, 40(4), 1105-1112.

Pochard, F., Azoulay, E., \& Chevret, S. Lemaire, F., Hubert, P., Canoui, P., ...\& Schlemmer B; (2001). Symptoms of anxiety and depression in family members of intensive care unit patients: ethical hypothesis regarding decision-making capacity. Critical Care Medicine 29, 1893-1897.

Puntillo, K. A., Arai, S., Cohen, N. H., Gropper, M.A., Neuhouse, J., Paul, S.M., \& Miaskowski, C. (2010). Symptoms experienced by intensive care unit patients at high risk of dying 
Critical Care Medicine, 38(11), 2155-2160. doi: 10.1097/CCM.0b013e3181f267ee

Reisfield, G.M. \& Wilson, G.R. Prognostication in heart failure. Fast facts and concepts. \#143. Available at: http://www.eperc.mcw.edu/EPERC/FastFactsIndex/ff 143.htm.

Resar, R., Griffin, F.A., Haraden, C., \& Nolan, T.W. (2005). Using Care Bundles to Improve Health Care Quality. IHI Innovation Series white paper. Cambridge, Massachusetts: Institute for Healthcare Improvement. Retrieved from Institute of Healthcare Innovations website: http://www.IHI.org

Rogers, E. (2003). Diffusion of Innovations. New York: Free Press.

Rogers, E. (2004). A prospective and retrospecive look at the diffusion model. Journal of Health Communication 9, 13-19. doi:10.1080/10810730490271449

Rost, N.S., Smith, E.E., Chang, Y., Snider, R.W., Chanderraj, R., Schwab, K., ...\& Rosand, J. (2008). Prediction of functional outcome in patients with primary intracerebral hemorrhage: The FUNC score. Stroke. doi: 10.1161/STROKEAHA.107.512202

Scottish Intercollegiate Guidelines Network. (2008). Critical appraisal: Notes and checklist. [On line]. Retrieved from the Scottish Intercollegiate Guidelines Network webpage: www.sign.ac.uk/methodology/checklist.html.

Selecky, P.A., Eliasson, C.A., Hall, R.I., Schneider, R.F., Varkey, B., \& McCaffree, D.R. (2005). Palliative and end-of-life care for patients with cardiopulmonary diseases: American College of Chest Physicians position statement. Chest, 128 (3), 599-610.

Shirley, M. (2006). Evidence-based Practice: How nurse leaders can facilitate innovation. Nursing Administration Quarterly, 252-265.

Singer, P.A. Bowman, K.W. (2002). Quality end-of-life care: A global perspective. BMC Palliative Care, (1)4, doi: 10.1186/1472-684X-1-4 
Singer, P.A., Martin, D.K., \& Kelner, M. (1999). Quality end-of-life care: patients' perspectives. Journal of the American Medical Society, 281, 163-168. doi: 10.1001/jama.281.2.163

Steinhauser, K.E., Christakis, N.A., Clipp, E.C., McNeilly, M., McIntyre, L., \& Tulsky, J.A. (2000). Factors considered important at the end of life by patients, family, physicians, and other care providers. Journal of the American Medical Association (284)19, 24762482. doi: 10.1001/jama.284.19.2476

Stein-Parbury, J. \& McKinley, S. (2000). Patients' experience of being in an intensive care unit: A select literature review. American Journal of Critical Care. 920-927.

SUPPORT Principal Investigators (1995). The study to understand prognoses and preferences for outcomes and risks of treatments (SUPPORT). JAMA, 274(20), 1591-1598.

SUPPORT Principal Investigators. (1996). A controlled trial to improve care for seriously ill hospitalized patients. JAMA, 275(16):1232.

Thompson, C., McCaughan, D., Cullum, N., Sheldon, T., \& Raynor, P. (2001). Nurses, information use, and clinical decision making--the real world potential for evidencebased decisions in nursing. Journal of Advanced Nursing, 36, 11-22.

Truog, R. D., Campbell, M. L., Curtis, J., Haas, C. E., Luce, J. M., Rubenfeld, G. D., ...\& Kaufman, D. C. (2008). Recommendations for end-of-life care in the intensive care unit: A consensus statement by the American Academy of Critical Care Medicine. Critical Care Medicine, 36(3), 953-963. doi:10.1097/CCM.0B013E3181659096

Vázquez, M., Pardavila, M., Lucia, M., Aguado, Y., Margall, M., \& Asiain, M. (2011). Pain assessment in turning procedures for patients with invasive mechanical ventilation. Nursing In Critical Care, 16(4), 178-185. doi:10.1111/j.1478-5153.2011.00436.x 
West Virginia Center for End-of-Life Care. (2011). West Virginia among leaders in advance directives. Retrieved from http://www.wvendoflife.org/News/2010-Survey-data

West Virginia Center for End-of-Life Care. (2013). WV Center for End of Life Care March 2013 tracking survey. Retrieved from: http://www.wvendoflife.org/MediaLibraries/WVCEOLC/Media/other/EOL-Report-2013FINAL.pdf

West Virginia Department of Health and Human Services. (2005). West Virginia Retrieved from the West Virginia Department of Health and Human Services website: http://www.wvdhhr.org/bph/hsc/pubs/vital/2005/vs_31.html

West Virginia Department of Health and Human Services. (2009). West Virginia vital statistics 2009. Available at: http://www.wvdhhr.org/bph/hsc/pubs/vital/2009/2009Vital.pdf

West Virginia Department of Health and Human Services. (2010). West Virginia Vital Statistics, 2010, West Virginia Bureau for Public Health, Health Statistics Center, sixty-fourth annual report. Retrieved from: http://www.wvdhhr.org/bph/hsc/pubs/vital/2010/2010Vital.pdf

WVU Healthcare. (2011, July 25). WVU Healthcare Sets Mission and Vision, and Adopts Strategic Plan. Retrieved from WVU Healthcare website: http://wvuhealthcare.com/IWVUH/08-15-11/July-Strategic-Plan-Memo-Final.pdf

WVU Healthcare (2011, March). Nursing research. Administrative Nursing Policy and Procedure (1), Retrieved from WVU Healthcare Intranet - public access denied.

White, D. B. \& Luce, J. M. (2004). Palliative care in the intensive care unit: Barriers, advances, and unmet needs. Critical Care Clinicians (20); 329-344 
World Health Organization. (2012). WHO definition of palliative care. Retrieved from World Health Organization website: http://www.who.int/cancer/palliative/definition/en/ 